\title{
MONOTONICITY FORMULAE AND HOLOMORPHICITY OF HARMONIC MAPS BETWEEN KÄHLER MANIFOLDS
}

\author{
YUXIN DONG
}

\begin{abstract}
In this paper, we introduce the stress-energy tensors of the partial energies $E^{\prime}(f)$ and $E^{\prime \prime}(f)$ of maps between Kähler manifolds. Assuming the domain manifolds poss some special exhaustion functions, we use these stress-energy tensors to establish some monotonicity formulae of the partial energies of pluriharmonic maps into any Kähler manifolds and harmonic maps into Kähler manifolds with strongly semi-negative curvature respectively. These monotonicity inequalities enable us to derive some holomorphicity and Liouville type results for these pluriharmonic maps and harmonic maps. We also use the stress-energy tensors to investigate the holomorphic extension problem of $C R$ maps.
\end{abstract}

\section{Introduction}

In 1980, Baird and Eells [BE] introduced the stress-energy tensor for maps between Riemannian manifolds, which unifies various results on harmonic maps. Following $[\mathrm{BE}]$, Sealey [Se] introduced the stress-energy tensor for $p$-forms with values in vector bundles and established some vanishing theorems for harmonic $p$-forms. Since then, the stress-energy tensors have become a useful tool for investigating the energy behavior of vector bundle valued $p$-forms in various problems. Recently the authors in $[\mathrm{DW}]$ presented a unified method to establish monotonicity formulae for $p$-forms with values in vector bundles by means of the stress-energy tensors of various energy functionals in geometry and physics. Since the stress-energy tensors are 2-tensor fields, we may get 1-forms by contracting them with vector fields. The divergence of these 1-forms then leads to a fundamental integral formula, which is naturally linked to conservation laws. The integral formula turns out to be a useful tool for establishing monotonicity formulae of the energies, provided that the $p$-forms satisfy the conservation laws and the radial curvatures of the domain manifolds satisfy some pinching conditions. Besides their possible applications in regularity problems, these monotonicity formulae enable us not only to deduce some vanishing theorems for $p$-forms under suitable growth conditions, but also to investigate constant Dirichlet boundary value problems for 1 -forms. In [DW], the authors mainly used the distance

1991 Mathematics Subject Classification. Primary: 53C43, 53C55, 32L20.

Key words and phrases. stress energy tensor, conservation law, monotonicity formula, harmonic map, pluriharmonic map, holomorphic map.

*Supported by NSFC grant No 10971029, and NSFC-NSF grant No 1081112053 
function of a complete Riemannian manifold to construct the vector field in the integral formula. The Hessian of the distance function appears naturally in the integral formula. Consequently they used Hessian comparison theorems and coarea formula to obtain their results. For the purposes of this paper, we will consider more general exhaustion functions on domain manifolds to construct vector fields in applying the integral formula. Assuming that the domain manifolds poss some suitable exhaustion functions, we may also establish some monotonicity formulae for the $p$-forms which satisfy the conservation laws (see Proposition 1.2 in $\S 1$ ).

In [Si1,2], Siu studied the holomorphicity of harmonic maps from compact Kähler manifolds into compact Kähler manifolds with strongly negative curvature or compact quotients of irreducible symmetric bounded domains. The basic discovery of Siu was a $\partial \bar{\partial}$-Bochner formula for harmonic maps which does not involve the Ricci curvature tensor of the domains (this is where Kählerianity of the domains enters). Using his modified Bochner formula and integration by parts, he proved a vanishing theorem which implies that the harmonic maps in question are actually pluriharmonic and some curvature terms of the pull-back complexified tangent bundles vanish. The vanishing curvature terms, under the assumption of sufficiently high rank, forces the maps to be either holomorphic or anti-holomorphic. Later, Sampson [Sa] extended Siu's technique to treat harmonic maps of compact Kähler manifolds to more general targets. In particular, he showed that harmonic maps from compact Kähler manifolds to Riemannian manifolds with nonpositive Hermitian curvature are pluriharmonic too. In [Li] and [PRS], the authors generalized Sampson's pluriharmonicity result to noncompact setting by assuming some growth conditions on energy of the harmonic maps. On the other hand, some authors have investigated the holomorphicity or pluriharmonicity of stable harmonic maps from compact Kähler manifolds (cf. [SY], $[\mathrm{Ud}],[\mathrm{OU}])$. We refer the reader to $[\mathrm{To}]$ for other related progress not mentioned here.

In this paper, we investigate harmonic maps between complete Kähler manifolds. When the domain Kähler manifold is complete (noncompact), the idea of the $\partial \bar{\partial}$ Bochner technique in [Si1,2] together with the integration by parts does not work any more and the holomorphicity problem of the harmonic map is largely unknown. Notice that for a smooth map $f: M \rightarrow N$ between two Kähler manifolds, one may introduce two 1-forms $\sigma$ and $\tau$ with values in $f^{-1} T N$ whose vanishing characterizes the holomorphicity and anti-holomorphicity of the map respectively. Actually $\sigma=$ $\bar{\partial} f+\partial \bar{f}$ and $\tau=\partial f+\overline{\partial f}$ if $\sigma$ and $\tau$ are complexified (see $\S 2$ ). The $L^{2}$ energies of $\sigma$ and $\tau$ are just the partial energies $E^{\prime \prime}(f)$ and $E^{\prime}(f)$ respectively. Therefore we have the stress-energy tensors $S_{\sigma}$ and $S_{\tau}$ corresponding to $\sigma$ and $\tau$ respectively. It is natural to attempt to apply $S_{\sigma}$ and $S_{\tau}$ to investigate the energy behavior of the partial energies and obtain vanishing theorems for $\sigma$ and $\tau$. For this purpose, we assume that the domain manifolds poss some suitable exhaustion functions. The advantages of using more general exhaustion functions instead of the distance functions in establishing monotonicity and vanishing results are that one may not only relax the curvature conditions on the domain manifolds but also has more choices for constructing suitable vector fields in the integral formula. Fortunately some classes of complex manifolds poss the required exhaustion functions. As the results of this method, we obtain the pluriharmonicity of a harmonic map, the monotonicity formulae for partial energies of a pluriharmonic map or a harmonic map, the holomorphicity and constancy of a 
pluriharmonic map or a harmonic map, and the holomorphic extensions of $C R$ maps, etc.

Our method is based on the formulae (1.12), (1.13) for $\sigma$ and $\tau$, and computing $\operatorname{div}\left(S_{\sigma}\right)$ and $\operatorname{div}\left(S_{\tau}\right)$. There are two 1-forms $\gamma$ and $\rho$ arising naturally in $\operatorname{div}\left(S_{\sigma}\right)$ and $\operatorname{div}\left(S_{\tau}\right)$. Then we derive the divergence formulae of $\gamma$ and $\rho$, which are Weitzenböcktype formulae involving only the square norm of the $(1,1)$-part of the second fundamental form $\nabla d f$ and the curvature of the target manifold. Assuming the domain Kähler manifold posses some exhaustion function, these two divergence formulae enable us to prove that a harmonic map into a Kähler manifold with strongly semi-negative curvature is pluriharmonic if either $|\bar{\partial} f|^{2}$ or $|\partial f|^{2}$ satisfies some nonintegrability condition (see Theorem 3.6). It follows that if one of the partial energies has growth order at most 2 (with respect to the exhaustion function), then the harmonic map is pluriharmonic (see Corollary 3.7). In this way, we generalize Siu's pluriharmonicity result to the non-compact setting. While the authors in [PRS] considered more general targets in their pluriharmonicity result by assuming the nonintegrability condition on the energy density, we only assume the nonintegrability condition on one of the partial energy densities to derive the pluriharmonicity.

Next we investigate the monotonicity and holomorphicity of harmonic maps between Kähler manifolds. First, we show that if $f: M \rightarrow N$ is pluriharmonic, then $\sigma$ and $\tau$ satisfy the conservation laws, that is, $\operatorname{div} S_{\sigma}=\operatorname{div} S_{\tau}=0$. Assuming $M$ posses a special exhaustion function (see (4.1),(4.2) and (4.3)), it turns out that the conditions of Proposition 1.2 are satisfied in this case. Hence we are able to establish the monotonicity formulae for the partial energies of the pluriharmonic map (see Theorem 4.3). It follows from the known comparison theorems that the distance functions of some complete Kähler manifolds become special exhaustion functions if their radial curvatures have some suitable upper bounds (see Lemma 4.6). This leads to the monotonicity formulae of pluriharmonic maps from these complete Kähler manifolds. Remarkably no curvature conditions are assumed on the targets for these results on pluriharmonic maps. When a harmonic map between two Kähler manifolds is not pluriharmonic, $\sigma$ and $\tau$ don't satisfy the conservation laws in general. Due to this non-conservativity, we can not apply Proposition 1.2 directly to $\sigma$ and $\tau$. However, if the target Kähler manifold has strongly semi-negative curvature, the fundamental integral formulae related to the stress-energy tensors are still applicable, because both $\operatorname{div}\left(S_{\sigma}\right)$ and $\operatorname{div}\left(S_{\tau}\right)$ contracted with suitable vector fields have some non-negativity (see Lemma 4.9). Therefore one may establish the monotonicity formulae of harmonic maps from certain Kähler manifolds to Kähler manifolds with strongly semi-negative curvature too (see Theorem 4.10). Besides the global monotonicity formulae, we also obtain some local monotonicity formulae for partial energies of pluriharmonic maps into Kähler manifolds or harmonic maps into Kähler manifolds with strongly seminegative curvature. Here, by "local" we mean that the monotonicity formulae hold either in a neighborhood of a point or outside a compact subset. Notice that the authors in [DW] assumed some curvature pinching conditions to establish monotonicity formulae for general $p$-forms. However, the special properties (2.12) of $\sigma$ and $\tau$ enable us not only to establish the monotonicity formulae on domain Kähler manifolds whose curvatures only have some upper bounds, but also to deduce the monotonicity formulae outside a compact subset. All these monotonicity formulae imply immedi- 
ately the holomorpicity of the harmonic maps or pluriharmonic maps under suitable growth conditions on the partial energies. In particular, Liouville type theorems follow from suitable growth condition on the energy of the maps. We should mention that a somewhat related approach has been used by other authors, see e.g. [Ta 1,2]. However, he used a integral formula technique to estimate the energy of harmonic maps between Kähler manifolds (not the partial energies) and was forced to get only Liouville type results. Our method of using stress energy tensors seems to be easily operational and can also be used to simplify the arguments in [Ta1,2].

The classical Bochner theorem [Bo] asserts that if $f$ is a smooth CR function on the smooth connected boundary $\partial D$ of a bounded domain $D$ in $C^{m}$, then $f$ can be extended from $\partial D$ to $\bar{D}$ so that $f$ is holomorphic in $D$. The Bochner type holomorphic extension problem for maps between Kähler manifolds becomes a much harder problem, which has been studied by several authors. In [Si2], [Sh], [NS], [Wo] and $[\mathrm{CL}]$, the authors took a harmonic map approach by using Siu's $\partial \bar{\partial}-$ Bochner formula. The basic procedure for this problem is as follows: First, one may find a harmonic extension $f$ of the boundary map by the existence result of Hamilton [Ha] and Schoen [Sc]. Next, try to derive the holomorphcity of the harmonic extension.

Notice that the harmonic extension $f$ satisfies the tangential Cauchy-Riemann equation on $\partial D$ if and only if the 1 -form $\sigma$ annihilates any tangent vector in the holomorphic distribution $H$ of $\partial D$, that is, $\left.\sigma\right|_{H}=0$. In their generalization of KarcherWood theorem about constant Dirichlet boundary problem for harmonic maps $[\mathrm{KW}]$, the authors in $[\mathrm{DW}]$, using the stress-energy tensor and its related integral formula, proved that if a 1-form with values in a vector bundle satisfies the conservation law over a starlike smooth domain $\Omega$ in certain Riemannian manifold and annihilates any tangent vector of $\partial \Omega$, then the 1 -form vanishes on $\Omega$. We show that this method can also be applied to investigate the vanishing of $\sigma$, although it only annihilates vectors in a subbundle of $T(\partial D)$. Here (2.12) plays an important role too. Consequently we are able to give an alternative proof of the result in [CL](see Proposition 6.6) and obtain some other holomorphic extension results not included in [Si2], [Sh] and [CL] (see $\S 6$ for details).

This paper is organized as follows. In $\S 1$, we recall some basic notions and formulae, and then describe briefly the approach of [DW], but in a slightly generalized way. In $\S 2$, we introduce the stress-energy tensor $S_{\sigma}$ and $S_{\tau}$ corresponding to $\sigma$ and $\tau$ respectively. The relationship among the stress-energy tensor $S_{f}$ introduced by BairdEells $[\mathrm{BE}]$ and the stress-energy tensors $S_{\sigma}, S_{\tau}$ are discussed. In $\S 3$, we give some criteria for the pluriharmonicity of a harmonic map between Kähler manifolds. $\S 4$ and $\S 5$ are devoted to the monotonicity formulae and holomorphicity of a pluriharmonic map or a harmonic map between Kähler manifolds. Finally, in $\S 6$, we investigate the holomorphic extension problem of a CR boundary map.

\section{Monotonicity formulae and vanishing results of $p$-forms}

Let $(M, g)$ be a Riemannian manifold and $\xi: E \rightarrow M$ a Riemannian vector bundle over $M$ with a metric compatible connection $\nabla^{E}$. Let $A^{p}(\xi)$ denote the space of smooth $p$-forms on $M$ with values in the vector bundle $\xi: E \rightarrow M$, that is, $A^{p}(\xi)=$ $\Gamma\left(\Lambda^{p} T^{*} M \otimes E\right)$. The exterior covariant differentiation $d^{\nabla}: A^{p}(\xi) \rightarrow A^{p+1}(\xi)$ relative 
to the connection $\nabla^{E}$ is defined by (cf. [EL])

$$
\begin{aligned}
d^{\nabla} \omega\left(X_{1}, \ldots, X_{p+1}\right) & =\sum_{i=1}^{p+1}(-1)^{i+1} \nabla_{X_{i}}^{E}\left(\omega\left(X_{1}, \ldots, \widehat{X}_{i}, \ldots, X_{p+1}\right)\right) \\
& +\sum_{i<j}(-1)^{i+j} \omega\left(\left[X_{i}, X_{j}\right], X_{1}, \ldots, \widehat{X}_{i}, \ldots, \widehat{X}_{j}, \ldots, X_{p+1}\right)
\end{aligned}
$$

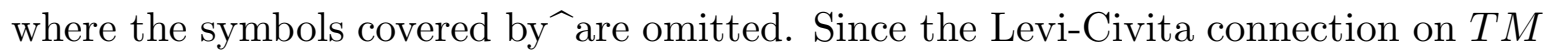
is torsion-free, we also have

$$
\left(d^{\nabla} \omega\right)\left(X_{1}, \ldots, X_{p+1}\right)=\sum_{i=1}^{p+1}(-1)^{i+1}\left(\nabla_{X_{i}} \omega\right)\left(X_{1}, \ldots, \widehat{X}_{i}, \ldots, X_{p+1}\right)
$$

The induced inner product on $\Lambda^{p} T_{x}^{*} M \otimes E_{x}$ is defined as follows:

$$
<\alpha, \beta>=\sum_{i_{1}<\cdots<i_{p}}<\alpha\left(e_{i_{1}}, \ldots, e_{i_{p}}\right), \beta\left(e_{i_{1}}, \ldots, e_{i_{p}}\right)>_{E_{x}}
$$

where $\left\{e_{1}, \ldots, e_{m}\right\}$ is an orthonormal basis of $T_{x} M, \forall x \in M$. Relative to the Riemannian structures of $E$ and $T M$, the codifferential operator $\delta^{\nabla}: A^{p}(\xi) \rightarrow A^{p-1}(\xi)$ is characterized as the adjoint of $d^{\nabla}$ via the formula:

$$
\int_{M}<d^{\nabla} \omega, \theta>d v_{g}=\int_{M}<\omega, \delta^{\nabla} \theta>d v_{g}
$$

where $\omega \in A^{p-1}(\xi), \theta \in A^{p}(\xi)$, one of which has compact support. Then

$$
\left(\delta^{\nabla} \theta\right)\left(X_{1}, \ldots, X_{p-1}\right)=-\sum_{i}\left(\nabla_{e_{i}} \theta\right)\left(e_{i}, X_{1}, \ldots, X_{p-1}\right)
$$

Let $f: M \rightarrow N$ be a smooth map between two Riemannian manifolds. The pull-back bundle $f^{-1} T N$ is endowed with the pull-back Riemannian structure. The differential $d f$ may be regarded as an element of $A^{1}\left(f^{-1} T N\right)$. The energy of $f$ is defined by

$$
E(f)=\frac{1}{2} \int_{M}|d f|^{2} d v_{g}
$$

In [BE], Baird-Eells introduced the stress-energy tensor $S_{f}$ associated with $E(f)$ as follows

$$
S_{f}=\frac{|d f|^{2}}{2} g-d f \odot d f
$$

where $d f \odot d f \in \Gamma\left(T^{*} M \otimes T^{*} M\right)$ is a symmetric tensor defined by

$$
(d f \odot d f)(X, Y)=<d f(X), d f(Y)>.
$$


Then they proved that a harmonic map satisfies the conservation law, that is, $d i v S_{f}=$ 0 .

In general, we may introduce the following energy functional for $\omega \in A^{p}(\xi)$

$$
F(\omega)=\int_{M}|\omega|^{2} d v_{g}
$$

The stress-energy tensor associated with $F$ is a symmetric 2-tensor field given by (cf. $[\mathrm{Se}],[\mathrm{Xi}],[\mathrm{Ba}])$ :

$$
S_{\omega}(X, Y)=\frac{|\omega|^{2}}{2} g(X, Y)-(\omega \odot \omega)(X, Y)
$$

for any $X, Y \in T M$, where $\omega \odot \omega$ denotes a 2 -tensor field defined by

$$
(\omega \odot \omega)(X, Y)=<i_{X} \omega, i_{Y} \omega>.
$$

Here $i_{X} \omega \in A^{p-1}(\xi)$ denotes the interior product by $X \in T M$, that is,

$$
\left(i_{X} \omega\right)\left(Y_{1}, \ldots, Y_{p-1}\right)=\omega\left(X, Y_{1}, \ldots, Y_{p-1}\right)
$$

for any $Y_{l} \in T M, 1 \leq l \leq p-1$.

For a 2-tensor field $T \in \Gamma\left(T^{*} M \otimes T^{*} M\right)$, its divergence $\operatorname{div} T \in \Gamma\left(T^{*} M\right)$ is defined by

$$
(\operatorname{div} T)(X)=\sum_{i}\left(\nabla_{e_{i}} T\right)\left(e_{i}, X\right), \quad \forall X \in T M
$$

where $\left\{e_{i}\right\}$ is an orthonormal basis of $T M$.

Lemma 1.1. (cf. [Se], [Xi], [Ba]) $\left(\operatorname{div} S_{\omega}\right)(X)=<\delta^{\nabla} \omega, i_{X} \omega>+<i_{X} d^{\nabla} \omega, \omega>$

Definition 1.1. $\omega \in A^{p}(\xi)$ is said to satisfy the conservation law if $S_{\omega}$ is divergence free, that is, $\operatorname{div} S_{\omega} \equiv 0$.

For a vector field $X$ on $M$, we denote by $\theta_{X}$ its dual one form, that is,

$$
\theta_{X}(Y)=g(X, Y), \quad \forall Y \in T M .
$$

The covariant derivative of $\theta_{X}$ gives a 2-tensor field $\nabla \theta_{X}$ :

$$
\left(\nabla \theta_{X}\right)(Y, Z)=\left(\nabla_{Z} \theta_{X}\right)(Y)=g\left(\nabla_{Z} X, Y\right), \quad \forall Y, Z \in T M .
$$

If $X=\nabla \psi$ is the gradient of some smooth function $\psi$ on $M$, then $\theta_{X}=d \psi$ and $\nabla \theta_{X}=\operatorname{Hess}(\psi)$.

For any vector field $X$ on $M$, a direct computation yields (cf. Lemma 2.4 of [DW]):

$$
\operatorname{div}\left(i_{X} S_{\omega}\right)=<S_{\omega}, \nabla \theta_{X}>+\left(\operatorname{div} S_{\omega}\right)(X) .
$$


Let $D$ be any bounded domain of $M$ with $C^{1}$-boundary. By (1.12) and using the divergence theorem, we immediately have the following integral formula (see also [Xi], $[\mathrm{DW}])$ :

$$
\int_{\partial D} S_{\omega}(X, \nu) d v_{\partial D}=\int_{D}\left[<S_{\omega}, \nabla \theta_{X}>+\left(\operatorname{div} S_{\omega}\right)(X)\right] d v_{g}
$$

where $\nu$ is the unit outward normal vector field along $\partial D$. In particular, if $\omega$ satisfies the conservation law, then

$$
\int_{\partial D} S_{\omega}(X, \nu)=\int_{D}<S_{\omega}, \nabla \theta_{X}>
$$

From now on, we often omit the volume elements in integral formulae for simplicity when the integral domains are clear.

Definition 1.2. A function $\Phi: M \rightarrow R$ is called an exhaustion function for a manifold $M$ if for every $t \in R$ the sublevel set $\{x \in M: \Phi(x)<t\}$ is relatively compact in $M$. The sublevel set $\{x \in M: \Phi(x)<t\}$ will be denoted by $B_{\Phi}(t)$.

Now we assume that $\Phi$ is a Lipschitz continuous exhaustion function for a Riemannian manifold $M$ satisfying the following conditions:

(1.15) $\Phi \geq 0$ and $\alpha=\sup _{x \in M}|\nabla \Phi|^{2}$ is finite;

(1.16) $\Psi=\Phi^{2}$ is of class $C^{\infty}$ and $\Psi$ has only discrete critical points.

It is a known fact that if a Riemannian manifold posses an exhaustion function $\Phi$ with the property (1.15), then it is complete. Actually let $r$ be the distance function relative a point $o \in M$ and $x_{k}$ a Cauchy sequence of $M$. The triangle inequality implies that $r\left(x_{k}\right) \leq C$ for some constant $C$. It follows that $\left|\Phi\left(x_{k}\right)\right| \leq|\Phi(o)|+\sqrt{\alpha} r\left(x_{k}\right) \leq$ $|\Phi(o)|+\sqrt{\alpha} C$. By the properness of $\Phi$, we see that the sequence $x_{k} \rightarrow x_{0} \in M$, that is, $M$ is complete.

Proposition 1.2. Let $M$ be a Riemannian manifold and let $\Phi$ be an exhaustion function with properties (1.15) and (1.16). Suppose $\xi: E \rightarrow M$ is a Riemannian vector bundle on $M$ and $\omega \in A^{p}(\xi)$ satisfies the conservation law. If there exists a positive constant $\beta$ such that

$$
<S_{\omega}, \operatorname{Hess}(\Psi)>\geq \beta|\omega|^{2}
$$

then

$$
\frac{1}{\rho_{1}^{\Lambda}} \int_{B_{\Phi}\left(\rho_{1}\right)}|\omega|^{2} \leq \frac{1}{\rho_{2}^{\Lambda}} \int_{B_{\Phi}\left(\rho_{2}\right)}|\omega|^{2}
$$

for any $0<\rho_{1} \leq \rho_{2}$, where $\Lambda=\beta / \alpha$. Furthermore, if $\int_{B_{\Phi}(t)}|\omega|^{2}=o\left(t^{\Lambda}\right)($ as $t \rightarrow \infty)$, then $\omega=0$.

Proof. Set $X=\frac{1}{2} \nabla(\Psi)=\Phi \nabla \Phi$. Obviously $\left.(\nabla \Psi)\right|_{\partial B_{\Phi}(t)}$ is an outward normal vector field along $\partial B_{\Phi}(t)$ if $t>0$ is a regular value of $\Psi$. By (1.8), we have

$$
\begin{aligned}
S_{\omega}(X, \nu) & =t<\nabla \Phi, \nu>\left[\frac{|\omega|^{2}}{2}-\left|i_{\nu} \omega\right|^{2}\right] \\
& \leq \frac{t \sqrt{\alpha}|\omega|^{2}}{2}
\end{aligned}
$$


on $\partial B_{\Phi}(t)$. It follows from $(1.14),(1.17)$ and (1.19) that

$$
t \sqrt{\alpha} \int_{\partial B_{\Phi}(t)} \frac{|\omega|^{2}}{2} \geq \frac{\beta}{2} \int_{B_{\Phi}(t)}|\omega|^{2} .
$$

By the co-area formula, we have

$$
\begin{aligned}
\frac{d}{d t} \int_{B_{\Phi}(t)}|\omega|^{2} & =\frac{d}{d t}\left\{\int_{0}^{t} \int_{\partial B_{\Phi}(s)} \frac{|\omega|^{2}}{|\nabla \Phi|}\right) d s \\
& =\int_{\partial B_{\Phi}(t)} \frac{|\omega|^{2}}{|\nabla \Phi|} \\
& \geq \frac{1}{\sqrt{\alpha}} \int_{\partial B_{\Phi}(t)}^{|\omega|^{2} .}
\end{aligned}
$$

Dividing both sides of (1.20) by $\alpha$, we obtain from(1.20) and (1.21) that

$$
t \frac{d}{d t} \int_{B_{\Phi}(t)}|\omega|^{2} \geq \Lambda \int_{B_{\Phi}(t)}|\omega|^{2}
$$

where $\Lambda=\beta / \alpha$. Hence we get

$$
\frac{d}{d t}\left(\frac{\int_{B_{\Phi}(t)}|\omega|^{2}}{t^{\Lambda}}\right) \geq 0
$$

This proposition follows immediately from integrating (1.22) on $\left[\rho_{1}, \rho_{2}\right]$.

Remark 1.1.

(a) A more general monotonicity inequality holds if one further assumes either $|\nabla \Phi|=$ const. or $\frac{|\omega|^{2}}{2}-\left|i_{\nu} \omega\right|^{2} \geq 0$ a.e. on $M$. In both cases, the first line of (1.19) yields

$$
S_{\omega}(X, \nu) \leq t \sqrt{\alpha}\left(\frac{|\omega|^{2}}{2}-\left|i_{\nu} \omega\right|^{2}\right)
$$

and thus

$$
t \sqrt{\alpha} \int_{\partial B_{\Phi}(t)}|\omega|^{2}-\beta \int_{B_{\Phi}(t)}|\omega|^{2} \geq 2 t \sqrt{\alpha} \int_{\partial B_{\Phi}(t)}\left|i_{\nu} \omega\right|^{2} .
$$

Similar arguments imply that

$$
\frac{d}{d t}\left(\frac{\int_{B_{\Phi}(t)}|\omega|^{2}}{t^{\Lambda}}\right) \geq \frac{2 t^{-\Lambda}}{\sqrt{\alpha}} \int_{\partial B_{\Phi}(t)}\left|i_{\nu} \omega\right|^{2}
$$

Hence

$$
\frac{1}{\rho_{2}^{\Lambda}} \int_{B_{\Phi}\left(\rho_{2}\right)}|\omega|^{2}-\frac{1}{\rho_{1}^{\Lambda}} \int_{B_{\Phi}\left(\rho_{1}\right)}|\omega|^{2} \geq \frac{2}{\sqrt{\alpha}} \int_{\rho_{1}}^{\rho_{2}} \frac{\int_{\partial B_{\Phi}(t)}\left|i_{\nu} \omega\right|^{2}}{t^{\Lambda}} d t
$$

for any $0<\rho_{1} \leq \rho_{2}$.

(b) The proof of Proposition 1.2 shows that the monotonicity formula still holds if one assumes $\int_{B_{\Phi}(t)} \operatorname{div} S_{\omega}(X) \geq 0$ for $t>0$ instead of assuming div $S_{\omega}=0$. We will consider these important non-conservative cases too.

We will apply Proposition 1.2 to investigate the monotonicity and holomorphicity of harmonic maps and pluriharmonic maps between Kähler manifolds. 


\section{The stress-energy tensors of $\bar{\partial} f$ and $\partial f$}

A Hermitian metric on a complex manifold $M$ is a Riemannian metric $g$ such that $g(J X, J Y)=g(X, Y), \forall X, Y \in T M$, where $J$ denotes the complex structure of $M$. We say that $(M, g)$ is Kähler if $J$ is parallel with respect to the Levi-Civita connection of $g$, that is, $\nabla J=0$.

We denote by $\langle\cdot, \cdot\rangle$ the (real) inner product of tensor bundles of $M$ induced by $g$. The complex extension of the inner product is still denoted by $\langle\cdot, \cdot\rangle$. Define the Hermitian inner product $\ll \cdot, \cdot \gg$ by

$$
\ll u, v \gg=<u, \bar{v}\rangle \text {. }
$$

Henceforth $\left(M^{m}, g\right)$ and $\left(N^{n}, h\right)$ will denote Kähler manifolds of complex dimensions $m$ and $n$ respectively. Let $f: M \rightarrow N$ be a smooth map from $M$ to $N$. The complex structure of $M$ (resp. $N$ ) gives a decompositions of $T M^{C}$ (resp. $T N^{C}$ ) into tangent vectors of type $(1,0)$ and type $(0,1)$. Then we have

$$
T M^{C}=T^{1,0} M \oplus T^{0,1} M, T N^{C}=T^{1,0} N \oplus T^{0,1} N .
$$

By restricting and projecting the complexified differential $d f: T M^{C} \rightarrow T N^{C}$ to the subbundles in (2.2), we have the following bundle maps (cf. also [Si2]):

$$
\begin{aligned}
& \partial f: T^{1,0} M \rightarrow T^{1,0} N, \quad \bar{\partial} f: T^{0,1} M \rightarrow T^{1,0} N \\
& \partial \bar{f}: T^{1,0} M \rightarrow T^{0,1} N, \quad \overline{\partial f}: T^{0,1} M \rightarrow T^{0,1} N .
\end{aligned}
$$

The energy functional of maps is defined by $E(f)=\frac{1}{2} \int_{M}|d f|^{2}$, where the energy density is

$$
\frac{1}{2}|d f|^{2}=\frac{1}{2} \sum_{j=1}^{m}\left\{<d f\left(e_{j}\right), d f\left(e_{j}\right)>+<d f\left(J e_{j}\right), d f\left(J e_{j}\right)>\right\}
$$

in terms of an orthonormal basis $\left\{e_{i}, J e_{i}\right\}_{i=1, \ldots, m} \in T M$. Set

$$
\eta_{j}=\frac{1}{\sqrt{2}}\left(e_{j}-i J e_{j}\right), \quad \eta_{\bar{j}}=\bar{\eta}_{j}=\frac{1}{\sqrt{2}}\left(e_{j}+i J e_{j}\right) .
$$

Then $\ll \eta_{j}, \eta_{k} \gg=\ll \eta_{\bar{j}}, \eta_{\bar{k}} \gg=\delta_{j k}$, that is $\left\{\eta_{j}\right\}_{j=1}^{m}$ (resp. $\left\{\eta_{\bar{j}}\right\}_{j=1}^{m}$ ) is a unitary basis of $T_{x}^{1,0} M$ (resp. $T_{x}^{0,1} M$ ). The partial energy densities of $f$ are defined by

$$
|\bar{\partial} f|^{2}=\sum_{j=1}^{m} \ll \bar{\partial} f\left(\bar{\eta}_{j}\right), \bar{\partial} f\left(\bar{\eta}_{j}\right) \gg, \quad|\partial f|^{2}=\sum_{j=1}^{m} \ll \partial f\left(\eta_{j}\right), \partial f\left(\eta_{j}\right) \gg .
$$

A direct computation gives

$$
\begin{aligned}
& |\bar{\partial} f|^{2}=\frac{1}{4} \sum_{j=1}^{m}\left\{<d f\left(e_{j}\right), d f\left(e_{j}\right)>+<d f\left(J e_{j}\right), d f\left(J e_{j}\right)>\right. \\
& \left.-2<d f\left(J e_{j}\right), J^{\prime} d f\left(e_{j}\right)>\right\}
\end{aligned}
$$


and

$$
\begin{aligned}
|\partial f|^{2}=\frac{1}{4} \sum_{j=1}^{m}\{ & <d f\left(e_{j}\right), d f\left(e_{j}\right)>+<d f\left(J e_{j}\right), d f\left(J e_{j}\right)> \\
& \left.+2<d f\left(J e_{j}\right), J^{\prime} d f\left(e_{j}\right)>\right\} .
\end{aligned}
$$

Hence we have

$$
E(f)=E^{\prime}(f)+E^{\prime \prime}(f)
$$

where $E^{\prime}(f)=\int_{M}|\partial f|^{2}$ and $E^{\prime \prime}(f)=\int_{M}|\bar{\partial} f|^{2}$ are the partial energies of $f$ respectively. The map $f: M \rightarrow N$ is called holomorphic (resp. anti-holomorphic) if

$$
d f \circ J=J \circ d f \quad\left(\text { resp. } d f \circ J=-J^{\prime} \circ d f\right)
$$

which is equivalent to $\bar{\partial} f=0$ (resp. $\partial f=0$ ).

For a smooth map $f: M \rightarrow N$, we introduce two 1 -forms $\sigma, \tau \in A^{1}\left(f^{-1} T N\right)$ as follows:

$$
\sigma(X)=\frac{d f(X)+J^{\prime} d f(J X)}{2}
$$

and

$$
\tau(X)=\frac{d f(X)-J^{\prime} d f(J X)}{2}
$$

for any $X \in T M$. Then (2.7) and (2.8) yield

$$
\sigma(J X)=\frac{d f(J X)-J^{\prime} d f(X)}{2}=-J^{\prime} \sigma(X)
$$

and

$$
\tau(J X)=\frac{d f(J X)+J^{\prime} d f(X)}{2}=J^{\prime} \tau(X)
$$

By complex extension, we may regard $\sigma$ and $\tau$ as sections of $T^{*} M^{C} \otimes f^{-1} T N^{C}$. By (2.9) and (2.10), we have

$$
\begin{array}{ll}
\sigma: T^{0,1} M \rightarrow T^{1,0} N, & \sigma: T^{1,0} M \rightarrow T^{0,1} N \\
\tau: T^{1,0} M \rightarrow T^{1,0} N, & \tau: T^{0,1} M \rightarrow T^{0,1} N
\end{array}
$$

and

$$
\begin{aligned}
& <\sigma(J X), \sigma(J Y)>=<\sigma(X), \sigma(Y)> \\
& <\tau(J X), \tau(J Y)>=<\tau(X), \tau(Y)>
\end{aligned}
$$


It turns out that (2.12) is important for studying the paritial energies of a map. By (2.7) and (2.8), we also derive

$$
\begin{aligned}
|\sigma|^{2}= & \sum_{j=1}^{m}\left[<\sigma\left(e_{j}\right), \sigma\left(e_{j}\right)>+<\sigma\left(J e_{j}\right), \sigma\left(J e_{j}\right)>\right] \\
= & \frac{1}{2} \sum_{j=1}^{m}\left[<d f\left(e_{j}\right), d f\left(e_{j}\right)>+<d f\left(J e_{j}\right), d f\left(J e_{j}\right)>\right. \\
& \left.\quad-2<d f\left(J e_{j}\right), J^{\prime} d f\left(e_{j}\right)>\right]
\end{aligned}
$$

and

$$
\begin{aligned}
|\tau|^{2}= & \sum_{j=1}^{m}\left[<\tau\left(e_{j}\right), \tau\left(e_{j}\right)>+<\tau\left(J e_{j}\right), \tau\left(J e_{j}\right)>\right] \\
= & \frac{1}{2} \sum_{j=1}^{m}\left[<d f\left(e_{j}\right), d f\left(e_{j}\right)>+<d f\left(J e_{j}\right), d f\left(J e_{j}\right)>\right. \\
& \left.+2<d f\left(J e_{j}\right), J^{\prime} d f\left(e_{j}\right)\right] .
\end{aligned}
$$

Therefore

$$
\begin{aligned}
& |\sigma|^{2}+|\tau|^{2}=|d f|^{2} \\
& |\sigma|^{2}=2|\bar{\partial} f|^{2}, \quad|\tau|^{2}=2|\partial f|^{2} .
\end{aligned}
$$

Recall that the map $f$ is said to be harmonic if it satisfies the Euler-Lagrange equation of the energy functional $E(f)$, that is,

$$
\sum_{A=1}^{2 m}(\nabla d f)\left(e_{A}, e_{A}\right)=2 \sum_{j=1}^{m}(\nabla d f)\left(\eta_{j}, \eta_{\bar{j}}\right)=0
$$

where $\nabla d f$ denotes the second fundamental form of $f$ ([cf. [EL]).

Lemma 2.1. If $f: M \rightarrow N$ is a harmonic map between Kähler manifolds, then $\delta^{\nabla} \sigma=\delta^{\nabla} \tau=0$.

Proof. Let $\left\{e_{A}\right\}_{A=1, \ldots, 2 m}=\left\{e_{i}, J e_{i}\right\}_{i=1, \ldots, m}$ be an orthonormal frame field around $p \in M$ such that $\left(\nabla_{e_{A}} e_{B}\right)_{p}=0$. Since $f$ is harmonic, we have

$$
\begin{aligned}
\delta^{\nabla} \sigma & =-\sum_{A=1}^{2 m}\left(\nabla_{e_{A}} \sigma\right)\left(e_{A}\right) \\
& =-\frac{1}{2} \sum_{A=1}^{2 m} \nabla_{e_{A}}\left[d f\left(e_{A}\right)+J^{\prime} d f\left(J e_{A}\right)\right] \\
& =-\frac{1}{2} J^{\prime}(\nabla d f)\left(J e_{A}, e_{A}\right) \\
& =-\frac{J^{\prime}}{2} \sum_{i=1}^{m}\left[(\nabla d f)\left(J e_{i}, e_{i}\right)-(\nabla d f)\left(e_{i}, J e_{i}\right)\right] \\
& =0 .
\end{aligned}
$$


Likewise we have $\delta^{\nabla} \tau \equiv 0$.

By definition, the stress-energy tensors of $\sigma$ and $\tau$ are given as follows:

$$
\begin{aligned}
& S_{\sigma}(X, Y)=\frac{|\sigma|^{2}}{2} g(X, Y)-\sigma \odot \sigma(X, Y) \\
& S_{\tau}(X, Y)=\frac{|\tau|^{2}}{2} g(X, Y)-\tau \odot \tau(X, Y) .
\end{aligned}
$$

Using (2.12), we obtain $S_{\sigma}(J X, J Y)=S_{\sigma}(X, Y)$ and $S_{\tau}(J X, J Y)=S_{\tau}(X, Y)$. This shows that $S_{\sigma}$ and $S_{\tau}$ are $(1,1)$-type tensor fields, according to the decomposition $T^{*} M^{C} \otimes T^{*} M^{C}=T^{*(2,0)} M \oplus T^{*(1,1)} M \oplus T^{*(0,2)} M$.

We hope to find the relationship among the three stress-energy tensors $S_{f}, S_{\sigma}$ and $S_{\tau}$. From (1.6), (2.13) and (2.15), we get

$$
S_{f}-\left[S_{\sigma}+S_{\tau}\right]=\frac{1}{2}[(d f J) \odot(d f J)-d f \odot d f] .
$$

Write $\Psi_{f}=\frac{1}{2}[(d f \circ J) \odot(d f \circ J)-d f \odot d f]$. For any two vector $Z, W \in T^{1,0} M$, we obtain

$$
\begin{aligned}
\Psi_{f}(Z, \bar{W}) & =\frac{1}{2}[h(i d f(Z),-i d f(\bar{W}))-h(d f(Z), d f(\bar{W}))] \\
& =0
\end{aligned}
$$

This implies

$$
S_{f}^{(1,1)}=S_{\sigma}+S_{\tau}, \quad \Psi_{f}=S_{f}^{(2,0)}+S_{f}^{(0,2)} .
$$

Definition 2.1. A map $f: M \rightarrow N$ is called pluriconformal if $J$ is isometry w.r.t. $d f \odot d f$, that is, $\Psi_{f} \equiv 0$.

For any $Z=X-i J X, W=Y-i J Y \in T^{1,0} M$, we have

$$
\begin{aligned}
\left(f^{*} h\right)(Z, W) & =(d f \odot d f)(X, Y)-(d f \odot d f)(J X, J Y) \\
& -i[(d f \odot d f)(X, J Y)+(d f \odot d f)(J X, Y)] .
\end{aligned}
$$

Therefore $f$ is pluriconformal if and only if $\left(f^{*} h\right)^{(2,0)}=0$ or equivalently $\left(f^{*} h\right)^{(0,2)}=$ 0 . Clearly \pm holomorphic maps are pluriconformal. However the converse is not true in general. By $(2.16), S_{f}=S_{\sigma}+S_{\tau}$ if and only if $f$ is pluriconformal. Note also that the notion of pluriconformal maps may be defined for maps from a Kähler manifold to a Riemannian manifold, that is, the target manifold is not necessarily a Kähler manifold.

Let $f: M \rightarrow N$ be a smooth map from a Kähler manifold. The complexified second fundamental form $\nabla d f$ of $f$, as a section of $T^{*} M^{C} \otimes T^{*} M^{C} \otimes f^{-1} T N^{C}$, may be decomposed as follows:

$$
\nabla d f=(\nabla d f)^{(2,0)}+(\nabla d f)^{(1,1)}+(\nabla d f)^{(0,2)} .
$$


Definition 2.2. A smooth map $f: M \rightarrow N$ from a Kähler manifold is called pluriharmonic if $(\nabla d f)^{(1,1)} \equiv 0$, that is,

$$
(\nabla d f)(Z, \bar{W})=0
$$

for any $Z, W \in T^{(1,0)} M$.

We should mention that the notion of pluriharmonic maps is well-defined for any smooth map $\varphi: M \rightarrow \widetilde{N}$ from a Kähler manifold to a Riemannian manifold $\widetilde{N}$. When $\widetilde{N}=R,(\nabla d \varphi)^{(1,1)}$ will be called the complex Hessian of $\varphi$ and denoted by $H(\varphi)$. Note that any pluriharmonic map is automatically harmonic, and any \pm holomorphic map between Kähler manifolds is pluriharmonic too. Clearly a smooth map between two Kähler manifolds is pluriharmonic if and only if its restriction to every holomorphic curve in $M$ is harmonic (cf. [Ra] for more general cases). The notion of pluriharmonic maps lies between those of harmonic and \pm holomorphic maps. There is no difference between harmonic and pluriharmonic for the case $\operatorname{dim}_{C} M=1$.

For any $Z=X_{1}-i J X_{1}, W=X_{2}-i J X_{2} \in T^{(1,0)} M$, we have

$$
\begin{aligned}
(\nabla d f)(Z, \bar{W})= & (\nabla d f)\left(X_{1}, X_{2}\right)+(\nabla d f)\left(J X_{1}, J X_{2}\right) \\
& +i\left[(\nabla d f)\left(X_{1}, J X_{2}\right)-(\nabla d f)\left(J X_{1}, X_{2}\right)\right] .
\end{aligned}
$$

This shows that (2.19) is equivalent to

$$
(\nabla d f)\left(X_{1}, X_{2}\right)+(\nabla d f)\left(J X_{1}, J X_{2}\right)=0 .
$$

and

$$
(\nabla d f)\left(X_{1}, J X_{2}\right)-(\nabla d f)\left(J X_{1}, X_{2}\right)=0 .
$$

for any $X_{1}, X_{2} \in T M$. Define a $2-$ form $\alpha \in A^{2}\left(f^{-1} T N\right)$ by

$$
\alpha\left(X_{1}, X_{2}\right)=(\nabla d f)\left(X_{1}, J X_{2}\right)-(\nabla d f)\left(J X_{1}, X_{2}\right) .
$$

for any $X_{1}, X_{2} \in T M$. By (2.22), we obtain $\alpha\left(J X_{1}, J X_{2}\right)=\alpha\left(X_{1}, X_{2}\right)$. Writing $X_{2}=-J \widetilde{X}_{2}$ in (2.21), we see that (2.20) and (2.21) are actually equivalent. Hence we have proved the following:

Lemma 2.2. A map $f: M \rightarrow N$ is pluriharmonic if and only if $f$ satisfies (2.20), or equivalently $f$ satisfies (2.21), that is, $\alpha \equiv 0$.

Let $f: M \rightarrow N$ be a smooth map between Kähler manifolds with $y=f(x), x \in$ $M$. We may choose normal orthnormal frame fields $\left\{e_{j}, J e_{j}\right\}_{j=1}^{m}$ and $\left\{\widetilde{e}_{\alpha}, J^{\prime} \widetilde{e}_{\alpha}\right\}_{\alpha=1}^{n}$ around $x$ and $y$ respectively. Set

$$
\begin{gathered}
\eta_{j}=\frac{1}{\sqrt{2}}\left(e_{j}-i J e_{j}\right), \quad \eta_{\bar{j}}=\bar{\eta}_{j}=\frac{1}{\sqrt{2}}\left(e_{j}+i J e_{j}\right) \\
\widetilde{\eta}_{\alpha}=\frac{1}{\sqrt{2}}\left(\widetilde{e}_{\alpha}-i J^{\prime} \widetilde{e}_{\alpha}\right), \quad \eta_{\bar{\alpha}}=\bar{\eta}_{\alpha}=\frac{1}{\sqrt{2}}\left(\widetilde{e}_{\alpha}+i J \widetilde{e}_{\alpha}\right) .
\end{gathered}
$$


Put $f_{j}=d f\left(\eta_{j}\right)$ and $f_{\bar{j}}=d f\left(\eta_{\bar{j}}\right)$. From $(2.2)$, we have

$$
\begin{aligned}
& f_{j}=f_{j}^{(1,0)}+f_{j}^{(0,1)}=(\partial f)\left(\eta_{j}\right)+(\partial \bar{f})\left(\eta_{j}\right) \\
& f_{\bar{j}}=f_{\bar{j}}^{(1,0)}+f_{\bar{j}}^{(0,1)}=(\bar{\partial} f)\left(\eta_{\bar{j}}\right)+(\overline{\partial f})\left(\eta_{\bar{j}}\right) .
\end{aligned}
$$

Using the frame field $\left\{\widetilde{\eta}_{\alpha}, \widetilde{\eta}_{\bar{\alpha}}\right\}_{\alpha=1, \ldots, n}$, we may write $f_{j}$ and $f_{\bar{j}}$ as follows:

$$
\begin{aligned}
& f_{j}=\sum_{\alpha}\left(f_{j}^{\alpha} \widetilde{\eta}_{\alpha}+f_{j}^{\bar{\alpha}} \widetilde{\eta}_{\bar{\alpha}}\right) \\
& f_{\bar{j}}=\sum_{\alpha}\left(f_{\bar{j}}^{\alpha} \widetilde{\eta}_{\alpha}+f_{\bar{j}}^{\bar{\alpha}} \widetilde{\eta}_{\bar{\alpha}}\right) .
\end{aligned}
$$

Let $\left\{\theta^{j}, \theta^{\bar{j}}\right\}_{j=1}^{m}$ and $\left\{\widetilde{\theta}^{\alpha}, \widetilde{\theta}^{\bar{\alpha}}\right\}_{\alpha=1}^{n}$ be the dual frame fields of $\left\{\eta_{j}, \eta_{\bar{j}}\right\}$ and $\left\{\widetilde{\eta}_{\alpha}, \widetilde{\eta}_{\bar{\alpha}}\right\}$ respectively. Hence we may express the complexified second fundamental form $\nabla d f$ as follows

$$
\begin{aligned}
\nabla d f= & \sum_{k, j, \alpha}\left\{f_{k j}^{\alpha} \theta^{k} \otimes \theta^{j} \otimes \widetilde{\eta}_{\alpha}+f_{k j}^{\bar{\alpha}} \theta^{k} \otimes \theta^{j} \otimes \widetilde{\eta}_{\bar{\alpha}}\right. \\
& +f_{\bar{k} j}^{\alpha} \theta^{\bar{k}} \otimes \theta^{j} \otimes \widetilde{\eta}_{\alpha}+f_{\overline{k j}}^{\bar{\alpha}} \theta^{\bar{k}} \otimes \theta^{j} \otimes \widetilde{\eta}_{\bar{\alpha}} \\
& +f_{k \bar{j}}^{\alpha} \theta^{k} \otimes \theta^{\bar{j}} \otimes \widetilde{\eta}_{\alpha}+f_{k \bar{j}}^{\bar{\alpha}} \theta^{k} \otimes \theta^{\bar{j}} \otimes \widetilde{\eta}_{\bar{\alpha}} \\
& \left.+f_{\overline{k j}}^{\alpha} \theta^{\bar{k}} \otimes \theta^{\bar{j}} \otimes \widetilde{\eta}_{\alpha}+f_{\overline{k j}}^{\bar{\alpha}} \theta^{\bar{k}} \otimes \theta^{\bar{j}} \otimes \widetilde{\eta}_{\bar{\alpha}}\right\} .
\end{aligned}
$$

Then

$$
\begin{array}{ll}
f_{k j}^{\alpha}=f_{j k}^{\alpha}, & f_{k \bar{j}}^{\alpha}=f_{\bar{j} k}^{\alpha} \\
f_{k j}^{\alpha}=f_{k j}^{\alpha}, & \frac{\bar{\alpha}}{f_{k j}^{\alpha}}=f_{\bar{k} j}^{\bar{\alpha}} .
\end{array}
$$

and

$$
\begin{aligned}
(\nabla d f)^{(1,1)} & =\sum_{k, j, \alpha}\left\{f_{k \bar{j}}^{\alpha} \theta^{k} \otimes \theta^{\bar{j}} \otimes \widetilde{\eta}_{\alpha}+f_{k \bar{j}}^{\bar{\alpha}} \theta^{k} \otimes \theta^{\bar{j}} \otimes \widetilde{\eta}_{\bar{\alpha}}\right. \\
& \left.+f_{\bar{k} j}^{\alpha} \theta^{\bar{k}} \otimes \theta^{j} \otimes \widetilde{\eta}_{\alpha}+f_{\bar{k} j}^{\bar{\alpha}} \theta^{\bar{k}} \otimes \theta^{j} \otimes \widetilde{\eta}_{\bar{\alpha}}\right\} .
\end{aligned}
$$

From (2.28), we get

$$
\left|(\nabla d f)^{(1,1)}\right|^{2}=4 \sum_{k, j, \alpha}\left|f_{k j}^{\alpha}\right|^{2}
$$

It follows that $f$ is pluriharmonic if and only if $f_{k \bar{j}}^{\alpha}=0,1 \leq k, j \leq m, 1 \leq \alpha \leq n$. 


\section{Pluriharmonicity of harmonic maps}

First we recall some curvature conditions introduced by Y.T. Siu [Si1] (cf. also [Sa], [To], [OU]). Let $\left(M^{m}, g\right)$ be a Kähler manifold of complex dimension $m$. The curvature tensor $R$ of $M$ is defined by

$$
R(X, Y) Z=\nabla_{X} \nabla_{Y} Z-\nabla_{Y} \nabla_{X} Z-\nabla_{[X, Y]} Z, \quad \forall X, Y, Z \in T M .
$$

We denote by $Q$ the curvature operator defined by $R$

$$
<Q(X \wedge Y), Z \wedge W>=<R(X, Y) W, Z>, \quad \forall X, Y, Z, W \in T M .
$$

The complex extension of $Q$ to $\wedge^{2} T M^{C}$ is also denoted by $Q$. By (2.1), we have

$$
\ll Q(X \wedge Y), Z \wedge W \gg=<Q(X \wedge Y), \overline{Z \wedge W}>, \quad \forall X, Y, Z, W \in T M^{C} .
$$

The Kähler identity of $M$ yields

$$
Q\left|\wedge^{(2,0)} T M=Q\right| \wedge^{(0,2)} T M=0 .
$$

Set

$$
Q^{(1,1)}=Q: \wedge^{(1,1)} T M \rightarrow \wedge^{(1,1)} T M .
$$

Definition 3.1 ([Si1] ). The curvature tensor of $(M, g)$ is said to be strongly negative (resp. strongly semi-negative) if

$$
\ll Q^{(1,1)}(\xi), \xi \gg=<Q^{(1,1)}(\xi), \bar{\xi}>\quad<0 \quad(\text { resp. } \leq 0)
$$

for any $\xi=(Z \wedge W)^{(1,1)} \neq 0, Z, W \in T M^{C}$.

Writing $Z=Z^{(1,0)}+Z^{(0,1)}, W=W^{(1,0)}+W^{(0,1)}$ in Definition 3.1, we get

$$
\xi=Z^{(1,0)} \wedge W^{(0,1)}-W^{(1,0)} \wedge Z^{(0,1)} .
$$

and thus

$$
\begin{aligned}
& \ll Q(Z \wedge W), Z \wedge W \gg=\ll Q\left(Z^{(1,0)} \wedge W^{(0,1)}-W^{(1,0)} \wedge Z^{(0,1)},\right. \\
& Z^{(1,0)} \wedge W^{(0,1)}-W^{(1,0)} \wedge Z^{(0,1)} \gg .
\end{aligned}
$$

In [Si1,Si2], Y.T. Siu showed the following result by his $\partial \bar{\partial}-$ Bochner formula:

Proposition 3.1 ([Si1]). Let $f: M \rightarrow N$ be a harmonic map from a compact Kähler manifold into a Kähler manifold with strongly semi-negative curvature. Then $f$ is a pluriharmonic map and

$$
\ll \widetilde{Q}\left(f_{j} \wedge f_{k}\right), f_{j} \wedge f_{k} \gg=0, \quad j, k=1, \ldots, m
$$


where $\widetilde{Q}$ denotes the curvature operator of $N$.

When $N$ is a Kähler manifold with strongly negative curvature or an irreducible symmetric bounded domain, Siu derived the holomorphicity of $f$ under further rank condition on $d f$ (cf. also Lemma 5.9 below).

We will extend Proposition 3.1 to the complete noncompact case. Suppose $f$ : $M^{m} \rightarrow N^{n}$ is a harmonic map between Kähler manifold. Let $\left\{\eta_{j}, \eta_{j}\right\}_{j=1}^{m}$ and $\left\{\widetilde{\eta}_{\alpha}, \widetilde{\eta}_{\bar{\alpha}}\right\}_{\alpha=1}^{n}$ be the normal unitary frame fields defined in $(2.27)$ and let $\left\{\theta^{j}, \theta^{\bar{j}}\right\}_{j=1}^{m}$ and $\left\{\widetilde{\theta}^{\alpha}, \widetilde{\theta}^{\bar{\alpha}}\right\}_{\alpha=1}^{n}$ be their dual frame fields respectively. By the definition of $\sigma$, we obtain

$$
\sigma\left(\eta_{j}\right)=\left[d f\left(\eta_{j}\right)\right]^{(0,1)}=\sum_{\alpha} f_{j}^{\bar{\alpha}} \widetilde{\eta}_{\bar{\alpha}} .
$$

The complex conjugate of $\sigma\left(\eta_{j}\right)$ gives

$$
\sigma\left(\eta_{\bar{j}}\right)=\sum_{\alpha} f_{\bar{j}}^{\alpha} \widetilde{\eta}_{\alpha}
$$

Hence

$$
\begin{aligned}
\sigma & =\partial \bar{f}+\bar{\partial} f \\
& =\sum_{j, \alpha}\left(f_{j}^{\bar{\alpha}} \theta^{j} \otimes \widetilde{\eta}_{\bar{\alpha}}+f_{\bar{j}}^{\alpha} \theta^{\bar{j}} \otimes \widetilde{\eta}_{\alpha}\right) .
\end{aligned}
$$

Similarly we have

$$
\begin{aligned}
\tau & =\partial f+\overline{\partial f} \\
& =\sum_{j, \alpha}\left(f_{j}^{\alpha} \theta^{j} \otimes \widetilde{\eta}_{\alpha}+f_{\bar{j}}^{\bar{\alpha}} \theta^{\bar{j}} \otimes \widetilde{\eta}_{\bar{\alpha}}\right) .
\end{aligned}
$$

The covariant derivative of $\sigma$ is given by

$$
\begin{aligned}
\nabla \sigma= & \sum_{j, k, \alpha}\left\{f_{j k}^{\bar{\alpha}} \theta^{j} \otimes \theta^{k} \otimes \widetilde{\eta}_{\bar{\alpha}}+f_{j \bar{k}}^{\bar{\alpha}} \theta^{j} \otimes \theta^{\bar{k}} \otimes \widetilde{\eta}_{\bar{\alpha}}\right. \\
& \left.+f_{\bar{j} k}^{\alpha} \theta^{\bar{j}} \otimes \theta^{k} \otimes \widetilde{\eta}_{\alpha}+f_{\overline{j k}}^{\alpha} \theta^{\bar{j}} \otimes \theta^{\bar{k}} \otimes \widetilde{\eta}_{\alpha}\right\} .
\end{aligned}
$$

By (1.2), we have

$$
\begin{aligned}
\left(d^{\nabla} \sigma\right)(X, Y) & =\left(\nabla_{X} \sigma\right)(Y)-\left(\nabla_{Y} \sigma\right)(X) \\
& =(\nabla \sigma)(Y ; X)-(\nabla \sigma)(X ; Y) .
\end{aligned}
$$

By (3.14), (3.15) and using (2.31), we get

$$
\begin{aligned}
d^{\nabla} \sigma= & \sum_{j, k, \alpha}\left\{f_{j k}^{\bar{\alpha}}\left(\theta^{k} \wedge \theta^{j}\right) \otimes \widetilde{\eta}_{\bar{\alpha}}+f_{j \bar{k}}^{\bar{\alpha}}\left(\theta^{\bar{k}} \wedge \theta^{j}\right) \otimes \widetilde{\eta}_{\bar{\alpha}}\right. \\
& \left.+f_{\bar{j} k}^{\alpha}\left(\theta^{k} \wedge \theta^{\bar{j}}\right) \otimes \widetilde{\eta}_{\alpha}+f_{\bar{j} k}^{\alpha}\left(\theta^{\bar{k}} \wedge \theta^{\bar{j}}\right) \otimes \widetilde{\eta}_{\alpha}\right\} \\
= & \sum_{j, k, \alpha}\left(f_{k \bar{j}}^{\alpha}\left(\theta^{k} \wedge \theta^{\bar{j}}\right) \otimes \widetilde{\eta}_{\alpha}-f_{j \bar{k}}^{\bar{\alpha}}\left(\theta^{j} \wedge \theta^{\bar{k}}\right) \otimes \widetilde{\eta}_{\bar{\alpha}}\right) \\
= & \sum_{k, j, \alpha}\left(f_{k \bar{j}}^{\alpha}\left(\theta^{k} \wedge \theta^{\bar{j}}\right) \otimes \widetilde{\eta}_{\alpha}-f_{k \bar{j}}^{\bar{\alpha}}\left(\theta^{k} \wedge \theta^{\bar{j}}\right) \otimes \widetilde{\eta}_{\bar{\alpha}}\right) .
\end{aligned}
$$


By (2.8) and (2.9), we have $d f=\sigma+\tau$. Since $d^{\nabla} d f=0$, it follows from (3.16) that

$$
d^{\nabla} \tau=-d^{\nabla} \sigma=\sum_{k, j, \alpha}\left(-f_{k \bar{j}}^{\alpha}\left(\theta^{k} \wedge \theta^{\bar{j}}\right) \otimes \widetilde{\eta}_{\alpha}+f_{k \bar{j}}^{\bar{\alpha}}\left(\theta^{k} \wedge \theta^{\bar{j}}\right) \otimes \widetilde{\eta}_{\bar{\alpha}}\right)
$$

From (3.12) and (3.16), we obtain

$$
\begin{aligned}
& <\left(d^{\nabla} \sigma\right)\left(\eta_{k}, e_{A}\right), \sigma\left(e_{A}\right)> \\
& =<\left(d^{\nabla} \sigma\right)\left(\eta_{k}, \eta_{j}\right), \sigma\left(\eta_{\bar{j}}\right)>+<\left(d^{\nabla} \sigma\right)\left(\eta_{k}, \eta_{\bar{j}}\right), \sigma\left(\eta_{j}\right)> \\
& =\sum_{j, \alpha} f_{k \bar{j}}^{\alpha} f_{j}^{\bar{\alpha}}
\end{aligned}
$$

and

$$
<\left(d^{\nabla} \sigma\right)\left(\eta_{\bar{k}}, e_{A}\right), \sigma\left(e_{A}\right)>=\sum_{j, \alpha} f_{\bar{k} j}^{\bar{\alpha}} f_{\bar{j}}^{\alpha}
$$

Define a 1-form $\gamma$ as follows

$$
\gamma(X)=\sum_{A}<\left(d^{\nabla} \sigma\right)\left(X, e_{A}\right), \sigma\left(e_{A}\right)>
$$

By (3.18) and (3.19), we obtain

$$
\begin{aligned}
\gamma & =\sum_{k}\left[<\left(d^{\nabla} \sigma\right)\left(\eta_{k}, e_{A}\right), \sigma\left(e_{A}\right)>\theta^{k}+<\left(d^{\nabla} \sigma\right)\left(\eta_{\bar{k}}, e_{A}\right), \sigma\left(e_{A}\right)>\theta^{\bar{k}}\right] \\
& =\sum_{k}\left[\left(\sum_{j, \alpha} f_{k \bar{j}}^{\alpha} f_{j}^{\bar{\alpha}}\right) \theta^{k}+\sum_{j, \alpha}\left(f_{\bar{k} j}^{\bar{\alpha}} f_{\bar{j}}^{\alpha}\right) \theta^{\bar{k}}\right] .
\end{aligned}
$$

Consequently we get

$$
\begin{aligned}
\operatorname{div}(\gamma) & =\sum_{k}\left[\left(\sum_{j, \alpha} f_{k \bar{j}}^{\alpha} f_{j}^{\bar{\alpha}}\right)_{\bar{k}}+\left(\sum_{j, \alpha}\left(f_{\bar{k} j}^{\bar{\alpha}} f_{\bar{j}}^{\alpha}\right)\right)_{k}\right] \\
& =2 \sum_{k, j, \alpha}\left|f_{k \bar{j}}^{\alpha}\right|^{2}+\sum_{k, j, \alpha}\left(f_{k \overline{j k}}^{\alpha} f_{j}^{\bar{\alpha}}+f_{\bar{k} j k}^{\bar{\alpha}} f_{\bar{j}}^{\alpha}\right)
\end{aligned}
$$

By Ricci identity and Kähler identity, we have

$$
\left(f_{k \bar{j} k}^{\alpha}-f_{k \bar{k} \bar{j}}^{\alpha}\right) \widetilde{\eta}_{\alpha}=-f_{k}^{\beta} \widetilde{R}_{\beta}^{\alpha}\left(f_{\bar{j}}, f_{\bar{k}}\right) \widetilde{\eta}_{\alpha}
$$

where $\widetilde{R}$ denotes the curvature tensor of $N$. From (2.29) and (3.23), it follows that

$$
\begin{gathered}
f_{k \overline{j k}}^{\alpha}-f_{k \overline{k j}}^{\alpha}=-f_{k}^{\beta} \widetilde{R}_{\beta \gamma \bar{\delta}}^{\alpha} f_{\frac{j}{\gamma}}^{\alpha \bar{\delta}}-f_{k}^{\beta} \widetilde{R}_{\beta \bar{\gamma} \delta}^{\alpha} f_{\bar{j}}^{\bar{\gamma}} f_{\bar{k}}^{\delta} \\
f_{\bar{k} j k}^{\bar{\alpha}}-f_{\bar{k} k j}^{\bar{\alpha}}=-f_{\bar{k}}^{\bar{\beta}} \widetilde{R}_{\bar{\beta} \bar{\gamma} \delta}^{\bar{\alpha}} f_{j}^{\bar{\gamma}} f_{k}^{\delta}-f_{\bar{k}}^{\bar{\beta}} \widetilde{R}_{\bar{\beta} \gamma \bar{\delta}}^{\bar{\alpha}} f_{j}^{\gamma} f_{k}^{\bar{\delta}} . \\
17
\end{gathered}
$$


Hence

$$
\begin{aligned}
& \operatorname{div}(\gamma) \\
= & 2 \sum_{k, j, \alpha}\left|f_{k \bar{j}}^{\alpha}\right|^{2}-\sum_{k, j, \alpha} \widetilde{R}_{\beta \gamma \bar{\delta}}^{\alpha} f_{j}^{\bar{\alpha}} f_{k}^{\beta} f_{\bar{j}}^{\gamma} f_{\bar{k}}^{\bar{\delta}}-\sum_{k, j, \alpha} \widetilde{R}_{\beta \bar{\gamma} \delta}^{\alpha} f_{j}^{\bar{\alpha}} f_{k}^{\beta} f_{\bar{j}}^{\bar{\gamma}} f_{\bar{k}}^{\delta} \\
& -\sum_{k, j} \widetilde{R}_{\bar{\beta} \bar{\gamma} \delta}^{\bar{\alpha}} f_{\bar{j}}^{\alpha} f_{\bar{k}}^{\bar{\beta}} f_{j}^{\bar{\gamma}} f_{k}^{\delta}-\sum_{k, j} \widetilde{R}_{\bar{\beta} \gamma \bar{\delta}}^{\bar{\alpha}} f_{\bar{j}}^{\alpha} f_{\bar{k}}^{\bar{\beta}} f_{j}^{\gamma} f_{k}^{\bar{\delta}} \\
= & 2 \sum_{k, j, \alpha}\left|f_{k \bar{j}}^{\alpha}\right|^{2}-\sum_{k, j}\left\{\ll \widetilde{Q}\left(f_{j}^{(0,1)} \wedge f_{k}^{(1,0)}\right), f_{j}^{(0,1)} \wedge f_{k}^{(1,0)} \gg\right. \\
& +\ll \widetilde{Q}\left(f_{j}^{(0,1)} \wedge f_{k}^{(1,0)}\right), f_{j}^{(1,0)} \wedge f_{k}^{(0,1)} \gg+\ll \widetilde{Q}\left(f_{j}^{(0,1)} \wedge f_{k}^{(1,0)}\right), f_{j}^{(0,1)} \wedge f_{k}^{(1,0)} \gg \\
& \left.+\ll \widetilde{Q}\left(f_{j}^{(1,0)} \wedge f_{k}^{(0,1)}\right), f_{j}^{(0,1)} \wedge f_{k}^{(1,0)} \gg\right\} \\
= & 2 \sum_{k, j, \alpha}\left|f_{k \bar{j}}^{\alpha}\right|^{2}-\sum_{k, j}\left\{2 \ll \widetilde{Q}\left(f_{k}^{(1,0)} \wedge f_{j}^{(0,1)}\right), f_{k}^{(1,0)} \wedge f_{j}^{(0,1)} \gg\right. \\
& \left.-\ll \widetilde{Q}\left(f_{k}^{(1,0)} \wedge f_{j}^{(0,1)}\right), f_{j}^{(1,0)} \wedge f_{k}^{(0,1)} \gg-\ll \widetilde{Q}\left(f_{j}^{(1,0)} \wedge f_{k}^{(0,1)}\right), f_{k}^{(1,0)} \wedge f_{j}^{(0,1)} \gg\right\}
\end{aligned}
$$

that is,

$$
\operatorname{div}(\gamma)=2 \sum_{k, j, \alpha}\left|f_{k j}^{\alpha}\right|^{2}-\sum_{k, j} \ll \widetilde{Q}\left(f_{k} \wedge f_{j}\right), f_{k} \wedge f_{j} \gg .
$$

Likewise we define a 1 -form $\rho$ by

$$
\rho(X)=\sum_{A}<\left(d^{\nabla} \tau\right)\left(X, e_{A}\right), \tau\left(e_{A}\right)>.
$$

From (3.13) and (3.17), we get

$$
\begin{aligned}
\rho & =<d^{\nabla} \tau\left(\eta_{k}, e_{A}\right), \tau\left(e_{A}\right)>\theta^{k}+<d^{\nabla} \tau\left(\eta_{\bar{k}}, e_{A}\right), \tau\left(e_{A}\right)>\theta^{\bar{k}} \\
& =\sum_{k}\left[\left(\sum_{j, \alpha} f_{k \bar{j}}^{\bar{\alpha}} f_{j}^{\alpha}\right) \theta^{k}+\left(\sum_{j, \alpha}\left(f_{\bar{k} j}^{\alpha} f_{\bar{j}}^{\bar{\alpha}}\right) \theta^{\bar{k}}\right] .\right.
\end{aligned}
$$

A similar computation yields

$$
\operatorname{div}(\rho)=2 \sum_{k, j}\left|f_{k \bar{j}}^{\alpha}\right|^{2}-\sum_{k, j} \ll \widetilde{Q}\left(f_{k} \wedge f_{j}\right), f_{k} \wedge f_{j} \gg .
$$

Set $\widetilde{J}^{\prime}=-J^{\prime}$. Then we may define 1 -forms $\widetilde{\sigma}$ and $\widetilde{\tau}$ by (2.8), (2.9) and using the new complex structure $\widetilde{J}^{\prime}$ of $N$. It follows that $\sigma=\widetilde{\tau}$ and $\tau=\widetilde{\sigma}$. The right hand side of (3.24) is obviously independent of the choice of $J^{\prime}$ and $\widetilde{J}^{\prime}$. This explains the result $\operatorname{div}(\gamma)=\operatorname{div}(\rho)$. Clearly either (3.24) or (3.27) can be used to derive the pluriharmonicity and thus holomorphicity of a harmonic map between two compact Kähler manifolds. In this paper, we try to compute some terms related to the stressenergy tensors. These two formulae, combined with (1.13), will become useful tools for investigate harmonic maps between complete noncompact Kähler manifolds too. From (3.24) and (3.27), we immediately have 
Lemma 3.2. Let $f: M \rightarrow N$ be a harmonic map between two Kähler manifolds. If $N$ has strongly semi-negative curvature, then $\operatorname{div}(\gamma)=\operatorname{div}(\rho) \geq 0$.

Lemma 3.3. Let $M$ be a Riemannian manifold possing an exhaustion function $\Phi$ with the properties (1.15) and (1.16). Suppose $Z$ a vector field on $M$ such that

$$
\liminf _{R \rightarrow \infty} \frac{1}{R} \int_{B_{\Phi}(2 R)-B_{\Phi}(R)}\|Z\|=0 .
$$

If div $Z$ has an integral (that is, if either $(\operatorname{div} Z)^{+}$or (divZ $)^{-}$is integrable), then $\int_{M} \operatorname{div} Z=0$. In particular, if outside some compact set div $Z$ is everywhere $\geq 0$ (or $\leq 0)$ then $\int_{M} \operatorname{div} Z=0$.

Proof. This result was established in [Ka] for a complete Riemannian manifold with $\Phi=r$ (the distance function). The proof of Lemma 3.3 goes almost the same as the proof of the main theorem in $[\mathrm{Ka}]$, except for that one should use the general exhaustion function $\Phi$ to replace the distance function. We omit the detailed proof here.

Remark 3.1. It is clear that if $\liminf _{R \rightarrow \infty} \frac{1}{R} \int_{B_{\Phi}(R)}\|Z\|=0$, then $Z$ satisfies (3.28).

Proposition 3.4. Let $M$ be a Kähler manifold possing an exhaustion function $\Phi$ with the properties (1.15) and (1.16). Let $f: M \rightarrow N$ be a harmonic map from a Kähler manifold to a Kähler manifold with strongly semi-negative curvature. If $f$ satisfies either

$$
\liminf _{R \rightarrow \infty} \frac{1}{R} \int_{B_{\Phi}(2 R)-B_{\Phi}(R)}\|\gamma\|=0
$$

or

$$
\liminf _{R \rightarrow \infty} \frac{1}{R} \int_{B_{\Phi}(2 R)-B_{\Phi}(R)}\|\rho\|=0
$$

then $f$ is pluriharmonic.

Proof. Without loss of generality, we assume $f$ satisfies (3.29). By Lemma 3.2, $\operatorname{div}(\gamma) \geq 0$. Hence we obtain from Lemma 3.3 and (3.24) the following

$$
\begin{aligned}
\int_{M} \operatorname{div}(\gamma) & =2 \sum_{k, j, \alpha} \int_{M}\left|f_{k \bar{j}}^{\alpha}\right|^{2}-\sum_{k, j} \int_{M} \ll \widetilde{Q}\left(f_{k} \wedge f_{j}\right), f_{k} \wedge f_{j} \gg \\
& =0 .
\end{aligned}
$$

This proposition follows immediately from (3.31).

By (3.20) and (3.26), we get

$$
\begin{gathered}
\|\gamma\| \leq|\bar{\partial} f| \cdot\left|(\nabla d f)^{(1,1)}\right| \\
\|\rho\| \leq|\partial f| \cdot\left|(\nabla d f)^{(1,1)}\right| .
\end{gathered}
$$

Consequently we have 
Corollary 3.5. Let $f: M \rightarrow N$ be a harmonic map from a complete noncompact Kähler manifold to a Kähler manifold with strongly semi-negative curvature. If $f$ satisfies either

$$
\liminf _{r \rightarrow \infty} \frac{1}{r} \int_{B_{2 r}-B_{r}}|\bar{\partial} f| \cdot\left|(\nabla d f)^{(1,1)}\right|=0
$$

or

$$
\liminf _{r \rightarrow \infty} \frac{1}{r} \int_{B_{2 r}-B_{r}}|\partial f| \cdot\left|(\nabla d f)^{(1,1)}\right|=0
$$

then $f$ is pluriharmonic.

Theorem 3.6. Let $M$ be a Kähler manifold possing an exhaustion function $\Phi$ with the properties (1.15) and (1.16). Let $f:(M, g) \rightarrow(N, h)$ be a harmonic map into a Kähler manifold of strongly semi-negative curvature. If $f$ satisfies either

$$
\left(\int_{\partial B_{\Phi}(s)}|\bar{\partial} f|^{2}\right)^{-1} \notin L^{1}(+\infty)
$$

or

$$
\left(\int_{\partial B_{\Phi}(s)}|\partial f|^{2}\right)^{-1} \notin L^{1}(+\infty)
$$

then $f$ is pluriharmonic. In particular, $f$ satisfies (3.9), that is,

$$
\ll \widetilde{Q}\left(f_{j} \wedge f_{k}\right), f_{j} \wedge f_{k} \gg=0, \quad j, k=1, \ldots, m
$$

where $\widetilde{Q}$ denotes the curvature operator of $N$.

Proof. Without loss of generality, we assume that $f$ satisfies (3.32). It follows from (3.24) and the divergence theorem that

$$
\begin{aligned}
\int_{B_{\Phi}(t)} \sum_{k, j}\left|f_{k j}^{\alpha}\right|^{2} & \leq \frac{1}{2} \int_{B_{\Phi}(t)} \operatorname{div}(\gamma) \\
& =\frac{1}{2} \int_{\partial B_{\Phi}(t)} i_{\nu} \gamma
\end{aligned}
$$

where $\nu$ denotes the unit outward normal vector field of $\partial B_{\Phi}(t)$. By (3.21), we have

$$
\left|i_{\nu} \gamma\right| \leq 2|\bar{\partial} f|\left(\sum_{k, j, \alpha}\left|f_{k \bar{j}}^{\alpha}\right|^{2}\right)^{1 / 2}
$$

which yields

$$
\int_{\partial B_{\Phi}(t)} i_{\nu} \gamma \leq 2\left\{\int_{\partial B_{\Phi}(t)}|\bar{\partial} f|^{2}\right\}^{1 / 2}\left\{\int_{\partial B_{\Phi}(t)} \sum_{k, j, \alpha}\left|f_{k \bar{j}}^{\alpha}\right|^{2}\right\}^{1 / 2}
$$


Set

$$
\beta(t)=\int_{B_{\Phi}(t)} \sum_{k, j, \alpha}\left|f_{k \bar{j}}^{\alpha}\right|^{2}
$$

Then, by the co-area formula,

$$
\begin{aligned}
\beta^{\prime}(t) & =\frac{d}{d t} \int_{0}^{t}\left(\int_{\partial B_{\Phi}(s)} \frac{\sum_{k, j, \alpha}\left|f_{k \bar{j}}^{\alpha}\right|^{2}}{|\nabla \Phi|}\right) d s \\
& =\int_{\partial B_{\Phi}(t)} \frac{\sum_{k, j, \alpha}\left|f_{k \bar{j}}^{\alpha}\right|^{2}}{|\nabla \Phi|} \\
& \geq \frac{1}{\sqrt{\alpha}} \int_{\partial B_{\Phi}(t)} \sum_{k, j, \alpha}\left|f_{k j}^{\alpha}\right|^{2}
\end{aligned}
$$

where $\alpha$ is defined by (1.15). Putting (3.35), (3.36) and (3.37) together and squaring we finally get

$$
\beta(t)^{2} \leq \sqrt{\alpha}\left(\int_{\partial B_{\Phi}(t)}|\bar{\partial} f|^{2}\right) \beta^{\prime}(t)
$$

Suppose that $(\nabla d f)^{(1,1)} \neq 0$. Therefore there exists $t_{0}>0$ sufficiently large such that $\beta(t)>0$, for every $t \geq t_{0}$. Fix such an $t_{0}$. From (3.38) we deduce the following

$$
\sqrt{\alpha}\left(\beta\left(t_{0}\right)^{-1}-\beta(t)^{-1}\right) \geq \int_{t_{0}}^{t} \frac{d s}{\int_{\partial B_{\Phi}(s)}|\bar{\partial} f|^{2}}
$$

and letting $t \rightarrow+\infty$ we contradict (3.32). Hence $f$ is pluriharmonic. By definition, $\gamma \equiv 0$. Then (3.24) implies that $f$ satisfies (3.9).

Corollary 3.7. Let $M$ be a Kähler manifold possing an exhaustion function $\Phi$ with the properties (1.15) and (1.16). Let $f:(M, g) \rightarrow(N, h)$ be a harmonic map into a Kähler manifold with strongly semi-negative curvature. If $f$ satisfies either

$$
\int_{B_{\Phi}(R)}|\bar{\partial} f|^{2} \leq C R^{2}
$$

or

$$
\int_{B_{\Phi}(R)}|\partial f|^{2} \leq C R^{2}
$$

for any $R>0$ and some constant $C>0$, then $f$ is pluriharmonic. In particular, $f$ satisfies (3.34).

Proof. Set

$$
h(t)=\int_{\substack{B_{\Phi}(t) \\ 21}}|\bar{\partial} f|^{2}
$$


So, by co-area formula,

$$
h^{\prime}(t)=\int_{\partial B_{\Phi}(t)} \frac{|\bar{\partial} f|^{2}}{|\nabla \Phi|} \geq \frac{1}{\sqrt{\alpha}} \int_{\partial B_{\Phi}(t)}|\bar{\partial} f|^{2}
$$

Fix $t_{0}>0$, and let $t>t_{0}$. From Proposition 1.3 of [RS], we know that

$$
\int_{t_{0}}^{t}\left(\frac{s-t_{0}}{h(s)}\right) d s \leq C_{1} \int_{t_{0}}^{t} \frac{d s}{h^{\prime}(s)}
$$

for some constant $C_{1}>0$. Then (3.42) and (3.43) imply

$$
\int_{t_{0}}^{t} \frac{s-t_{0}}{\int_{B_{\Phi}(s)}|\bar{\partial} f|^{2}} d s \leq C_{1} \sqrt{\alpha} \int_{t_{0}}^{t} \frac{d s}{\int_{\partial B_{\Phi}(s)}|\bar{\partial} f|^{2}}
$$

Suppose $f$ satisfies (3.39). This implies

$$
\frac{s}{\int_{B_{\Phi}(s)}|\bar{\partial} f|^{2}} \notin L^{1}(+\infty)
$$

Thus we deduce from (3.44) and (3.45) that $f$ satisfies (3.32). Likewise we may prove that (3.40) implies (3.33). Hence we prove this corollary.

Remark 3.2. Suppose $f: M \rightarrow N$ is a harmonic map from a complete Kähler manifold to a Kähler manifold with strongly semi-negative curvature. We may take the distance function $r$ as the exhaustion function in Corollary 3.7. Therefore we have proved that if one of the partial energies of $f$ has growth order $\leq 2$ w.r.t. the distance function $r$, then $f$ is pluriharmonic. In particular, if either $E^{\prime}(f)<+\infty$ or $E^{\prime \prime}(f)<+\infty$, then $f$ is pluriharmonic. In [Li] and [PRS], the authors gave similar conditions about energy (not the partial energies) to derive the pluriharmonicity of a harmonic map from a complete Kähler manifold to a Riemannian manifold of non-positive Hermitian curvature. Obviously any criteria for pluriharmonicity is superfluous if $\operatorname{dim}_{C} M=1$. It would be interesting to know whether the growth order condition in Corollary 3.7 is optimal for ensuring the pluriharmonicity or not.

\section{Monotonicity formulae of harmonic maps}

\subsection{The case of pluriharmonic maps}

First we show that the 1 -forms $\sigma$ and $\tau$ defined in $\S 2$ satisfy the conservation laws if $f: M \rightarrow N$ is pluriharmonic.

Lemma 4.1. A map $f: M \rightarrow N$ between two Kähler manifolds is pluriharmonic if and only if the 1-form $\sigma \in A^{1}\left(f^{-1} T N\right)$ defined by (2.8) is closed or equivalently, the 1 -form $\tau \in A^{1}\left(f^{-1} T N\right)$ defined by (2.9) is closed. 
Proof. By definition, we have

$$
\begin{aligned}
& d^{\nabla} \sigma\left(X_{1}, X_{2}\right) \\
& =\nabla_{X_{1}} \sigma\left(X_{2}\right)-\nabla_{X_{2}} \sigma\left(X_{1}\right)-\sigma\left(\left[X_{1}, X_{2}\right]\right) \\
& =\frac{1}{2}\left\{(\nabla d f)\left(X_{2}, X_{1}\right)-(\nabla d f)\left(X_{1}, X_{2}\right)+J^{\prime}\left[(\nabla d f)\left(X_{1}, J X_{2}\right)-(\nabla d f)\left(X_{2}, J X_{1}\right)\right]\right\} \\
& =\frac{1}{2} J^{\prime}\left[(\nabla d f)\left(X_{1}, J X_{2}\right)-(\nabla d f)\left(X_{2}, J X_{1}\right)\right]
\end{aligned}
$$

Likewise we may get

$$
d^{\nabla} \tau\left(X_{1}, X_{2}\right)=-\frac{1}{2} J^{\prime}\left[(\nabla d f)\left(X_{1}, J X_{2}\right)-(\nabla d f)\left(X_{2}, J X_{1}\right)\right]
$$

It follows from Lemma 2.2 that $f$ is pluriharmonic iff $d^{\nabla} \sigma=0$ iff $d^{\nabla} \tau=0$.

Proposition 4.2. Suppose $f: M \rightarrow N$ is a pluriharmonic map between Kähler manifolds. Then $\operatorname{div} S_{\sigma}=\operatorname{div} S_{\tau}=0$.

Proof. This proposition follows immediately from Lemma 1.1, Lemma 2.1, Lemma 4.1.

Remark 4.1. Proposition 4.2 and (2.20) yield that $\operatorname{div} \Psi_{f}=0$ for a pluriharmonic map $f$, where $\Psi_{f}$ is the 2 -tensor field defined by (2.19).

From Proposition 1.2 and Proposition 4.2, we may deduce the energy monotonicity formulae for $\sigma$ and $\tau$ of pluriharmonic maps provided that $M$ posses suitable exhaustion functions. From now on we assume $\operatorname{dim}_{C} M=m \geq 2$, unless otherwise indicated.

Let $\Phi$ be a Lipschitz continuous function on $M^{m}$, which satisfies the following conditions (cf. also [Ta2]):

(4.1) $\Phi \geq 0$ and $\Phi$ is an exhaustion function of $M$, i.e., each sublevel set $B_{\Phi}(t):=$ $\{\Phi<t\}$ is relatively compact in $M$ for $t \geq 0$;

(4.2) $\Psi=\Phi^{2}$ is of class $C^{\infty}$ and $\Psi$ has only discrete critical points;

(4.3) The constant $k_{1}=\inf _{x \in M} \sum_{i=1}^{m-1} \varepsilon_{i}(x)$ is positive where $\varepsilon_{1} \leq \varepsilon_{2} \leq \cdots \leq$ $\varepsilon_{m}$ are the eigenvalues of the complex Hessian $H(\Psi)=\left(\Psi_{i \bar{j}}\right)$. The constant $k_{2}=$ $\sup _{x \in M}|\nabla \Phi|^{2}$ is finite. Set

$$
\lambda=k_{1} / k_{2}
$$

The function $\Phi$ with the properties (4.1), (4.2) and (4.3) will be called a special exhaustion function. Notice that (4.1) implies that $\operatorname{Im}(\Phi)=[0,+\infty)$. For our purpose, we only consider the unbounded exhaustion function for a complex manifold in this paper, although not all results need this assumption. In addition, the condition (4.3) for $\Psi$ is stronger than the condition for a function to be strictly $(m-1)$-plurisubharmonic (see $\S 6$ for some discussion about the notion of strict $(m-1)$-plurisubharmonicity). 
Theorem 4.3. Let $f: M \rightarrow N$ be a pluriharmonic map from a complete Kähler manifold to a Kähler manifold. Suppose $M$ posses a special exhaustion function $\Phi$ satisfying (4.1), (4,2) and (4.3). Then

$$
\frac{1}{\rho_{1}^{\lambda}} \int_{B_{\Phi}\left(\rho_{1}\right)}|\bar{\partial} f|^{2} \leq \frac{1}{\rho_{2}^{\lambda}} \int_{B_{\Phi}\left(\rho_{2}\right)}|\bar{\partial} f|^{2}
$$

and

$$
\frac{1}{\rho_{1}^{\lambda}} \int_{B_{\Phi}\left(\rho_{1}\right)}|\partial f|^{2} \leq \frac{1}{\rho_{2}^{\lambda}} \int_{B_{\Phi}\left(\rho_{2}\right)}|\partial f|^{2}
$$

for any $0<\rho_{1} \leq \rho_{2}$, where $\lambda$ is given by (4.4).

Proof. Take $X=\frac{1}{2} \nabla \Psi=\Phi \nabla \Phi$. Obviously $\left.(\nabla \Psi)\right|_{\partial B_{\Phi}(t)}$ is an outward normal vector field along $\partial B_{\Phi}(t)$ for a regular value $t>0$ of $\Phi$. Thus $\nabla \Psi=w(x) \nu$ on $\partial B_{\Phi}(t)$ with $w(x)>0$ for each point $x \in \partial B_{\Phi}(t)$, where $\nu$ denotes the unit outward normal vector field of $\partial B_{\Phi}(t)$. By the definition of $S_{\sigma}$, we have

$$
\begin{aligned}
S_{\sigma}(X, \nu) & =\frac{|\sigma|^{2}}{2}<X, \nu>_{g}-<\sigma(X), \sigma(\nu)>_{h} \\
& =t \frac{|\sigma|^{2}}{2}<\nabla \Phi, \nu>_{g}-\frac{w}{2}|\sigma(v)|_{h}^{2} \\
& \leq t \sqrt{k_{2}}|\bar{\partial} f|^{2}
\end{aligned}
$$

on $\partial B_{\Phi}(t)$ and

$$
\begin{aligned}
<S_{\sigma}, \nabla \theta_{X}> & =\frac{1}{2}<S_{\sigma}, \operatorname{Hess}(\Psi)> \\
& =\frac{1}{2}\left[\frac{|\sigma|^{2}}{2} \triangle_{g} \Psi-<\sigma \odot \sigma, \operatorname{Hess}(\Psi)>\right]
\end{aligned}
$$

where $\triangle_{g}$ denotes the Laplace-Beltrami operator on $M$. We choose a unitary basis $\left\{\eta_{i}=\left(e_{i}-i J e_{i}\right) / \sqrt{2}\right\}_{i=1, \ldots, m}$ at a point $p \in B_{\Phi}(t)$ such that

$$
\operatorname{Hess}(\Psi)\left(\eta_{i}, \bar{\eta}_{j}(p)\right)=\varepsilon_{i} \delta_{i j}
$$

which is equivalent to

$$
\begin{aligned}
& \operatorname{Hess}(\Psi)\left(e_{i}, e_{j}\right)+\operatorname{Hess}(\Psi)\left(J e_{i}, J e_{j}\right)=2 \varepsilon_{i} \delta_{i j} \\
& \operatorname{Hess}(\Psi)\left(e_{i}, J e_{j}\right)-\operatorname{Hess}(\Psi)\left(J e_{i}, e_{j}\right)=0
\end{aligned}
$$

Obviously $\left\{e_{i}, J e_{i}\right\}_{i=1, \ldots, m}$ is an orthonormal basis. Then (4.9) gives

$$
\triangle_{g} \Psi=2 \sum_{i=1}^{m} \varepsilon_{i}
$$


Using (2.12) and (4.9), we obtain

$$
\begin{aligned}
< & \sigma \odot \sigma, \operatorname{Hess}(\Psi)> \\
= & \sum_{i, j}\left\{<\sigma\left(e_{i}\right), \sigma\left(e_{j}\right)>\operatorname{Hess}(\Psi)\left(e_{i}, e_{j}\right)+<\sigma\left(J e_{i}\right), \sigma\left(J e_{j}\right)>\operatorname{Hess}(\Psi)\left(J e_{i}, J e_{j}\right)\right. \\
& \left.+<\sigma\left(e_{i}\right), \sigma\left(J e_{j}\right)>\operatorname{Hess}(\Psi)\left(e_{i}, J e_{j}\right)+<\sigma\left(J e_{i}\right), \sigma\left(e_{j}\right)>\operatorname{Hess}(\Psi)\left(J e_{i}, e_{j}\right)\right\} \\
= & \sum_{i, j}<\sigma\left(e_{i}\right), \sigma\left(e_{j}\right)>\left[\operatorname{Hess}(\Psi)\left(e_{i}, e_{j}\right)+\operatorname{Hess}(\Psi)\left(J e_{i}, J e_{j}\right)\right] \\
& +\sum_{i, j}<\sigma\left(e_{i}\right), \sigma\left(J e_{j}\right)>\left[\operatorname{Hess}(\Psi)\left(e_{i}, J e_{j}\right)-\operatorname{Hess}(\Psi)\left(J e_{i}, e_{j}\right)\right] \\
= & 2 \sum_{i=1}^{m}\left|\sigma\left(e_{i}\right)\right|^{2} \varepsilon_{i}
\end{aligned}
$$

From (4.8), (4.10) and (4.11), we get

$$
\begin{aligned}
<S_{\sigma}, \nabla \theta_{X}> & =\frac{1}{2}\left\{\frac{|\sigma|^{2}}{2}\left(2 \sum_{j=1}^{m} \varepsilon_{j}\right)-2 \sum_{i=1}^{m}\left|\sigma\left(e_{i}\right)\right|^{2} \varepsilon_{i}\right\} \\
& \geq \frac{1}{2}\left\{|\sigma|^{2} \sum_{j=1}^{m} \varepsilon_{j}-2 \sum_{i=1}^{m}\left|\sigma\left(e_{i}\right)\right|^{2} \varepsilon_{m}\right\} \\
& \geq \frac{k_{1}|\sigma|^{2}}{2}=k_{1}|\bar{\partial} f|^{2}
\end{aligned}
$$

It follows from (1.14), (4.7) and (4.12) that

$$
\sqrt{k_{2}} t \int_{\partial B_{\Phi}(t)}|\bar{\partial} f|^{2} \geq k_{1} \int_{B_{\Phi}(t)}|\bar{\partial} f|^{2}
$$

The remaining arguments are similar to those in the proof of Proposition 1.2. Using the coarea formula yields, we get

$$
\frac{d}{d t} \int_{B_{\Phi}(t)}|\bar{\partial} f|^{2} \geq \frac{1}{\sqrt{k_{2}}} \int_{\partial B_{\Phi}(t)}|\bar{\partial} f|^{2}
$$

and thus

$$
\frac{\frac{d}{d t} \int_{B_{\Phi}(t)}|\bar{\partial} f|^{2}}{\int_{B_{\Phi}(t)}|\bar{\partial} f|^{2}} \geq \frac{\lambda}{t}
$$

for any $t>0$, where $\lambda=k_{1} / k_{2}$. By integration over $\left[\rho_{1}, \rho_{2}\right]$, we have

$$
\frac{1}{\rho_{1}^{\lambda}} \int_{B_{\Phi}\left(\rho_{1}\right)}|\bar{\partial} f|^{2} \leq \frac{1}{\rho_{2}^{\lambda}} \int_{B_{\Phi}\left(\rho_{2}\right)}|\bar{\partial} f|^{2}
$$

for any $0<\rho_{1} \leq \rho_{2}$. Similarly we can prove

$$
\frac{1}{\rho_{1}^{\lambda}} \int_{B_{\Phi}\left(\rho_{1}\right)}|\partial f|^{2} \leq \frac{1}{\rho_{2}^{\lambda}} \int_{B_{\Phi}\left(\rho_{2}\right)}|\partial f|^{2}
$$


for any $0<\rho_{1} \leq \rho_{2}$.

Remark 4.2.

(a) Suppose $\widehat{\lambda}$ is a positive number less than $\lambda$, that is, $0<\hat{\lambda}<\lambda$. Clearly we have the corresponding monotonicity formulae by replacing $\lambda$ by $\hat{\lambda}$ in (4.5) and (4.6). The larger the growth order $\lambda$ we get, the better the monotonicity formulae become.

(b) In [Ta2], K. Takegoshi derived similar monotonicty formulae for the energy of pluriharmonic maps. Here we establish monotonicity formulae for the partial energies.

Now we give some examples of Kähler manifolds which poss the special exhaustion functions (Some of them were also discussed in [Ta2] with somewhat different notations) .

\section{Example 4.1.}

(a) Let $C^{m}$ be an $m \geq 2$ complex Euclidean space with the canonical Kähler metric. Take $\Psi=\sum_{j=1}^{m} z_{j} z_{\bar{j}}=\|z\|^{2}$ and $\Phi=\|z\|$. Then $\left(\Psi_{i \bar{j}}\right)=2\left(\delta_{i j}\right)_{m \times m}$ where the complex Hessian is diagonalized w.r.t. $\left\{\eta_{j}=\frac{1}{\sqrt{2}}\left(e_{j}-i J e_{j}\right)\right\}_{j=1}^{m}$. By definition, we have $k_{1}=2 m-2, k_{2}=1$ and thus $\lambda=2 m-2$.

(b) Let $i: M^{m} \rightarrow C^{N}$ be an $m$-dimensional closed complex submanifold and $F$ : $C^{N} \rightarrow R$ be a smooth function on $C^{N}$. By the composition formula of maps (cf.[EL]), we have

$$
\operatorname{Hess}(F \circ i)(X, Y)=(H e s s F)(X, Y)+d F(B(X, Y))
$$

for any $X, Y \in T M$, which yields

$$
\operatorname{Hess}(F \circ i)\left(\eta_{i}, \bar{\eta}_{j}\right)=(\operatorname{HessF})\left(\eta_{i}, \bar{\eta}_{j}\right)+d F\left(B\left(\eta_{i}, \bar{\eta}_{j}\right)\right)
$$

where $\left\{\eta_{i}=\frac{1}{\sqrt{2}}\left(e_{i}-i J e_{i}\right)\right\}_{i=1}^{m}$ is any unitary frame tangent to $M$. Since $i(M)$ is a complex submanifold, we have $B\left(\eta_{i}, \bar{\eta}_{j}\right)=0$. Then

$$
\operatorname{Hess}(F \circ i)\left(\eta_{j}, \bar{\eta}_{k}\right)=(\operatorname{HessF})\left(\eta_{j}, \bar{\eta}_{k}\right)
$$

Now let $F=\|z\|^{2}=\sum_{A=1}^{N}\left|z_{A}\right|^{2}$ and set $\Psi=\Phi^{2}=i^{*}(F)=F \circ i$. Obviously $k_{1}=2(m-1)$ and $k_{2} \leq 1$. If necessary, translating the original point to a general position, we may assume that $F$ is a Morse function (cf. [Mi]). So $F$ has only discrete critical points. Then $\Phi$ is a special exhaustion function with $\lambda \geq 2 m-2$. Recall that every Stein manifold $M^{m}$ can be realized as a closed submanifold of $C^{N}$ by a proper holomorphic map $\psi: M^{m} \rightarrow C^{N}$. Thus a Stein manifold $M$ admits a special exhaustion $\Psi=\Phi^{2}$ with $\Phi=\psi^{*}(\|z\|)$ and $\lambda \geq 2 m-2$. It is known that every closed complex submanifold of a Stein manifold is a Stein manifold too. Therefore Stein manifolds provide us many examples of Kähler manifolds which poss special exhaustion functions.

Notice that the special exhaustion functions in (b) of example 4.1 are obtained from extrinsic distance functions. Next we will show that under suitable curvature conditions, the distance functions of Kähler manifolds are special exhaustion functions too. 
Lemma 4.4. Let $M^{m}$ be an $m$ dimensional complete Kähler manifold with a pole $x_{0}$. Let $\varepsilon_{1} \leq \varepsilon_{2} \leq \cdots \leq \varepsilon_{m}$ be the eigenvalues of the complex Hessian $\left(\left(r^{2}\right)_{i \bar{j}}\right)$, where $r$ is the distance function relative to $x_{0}$. Suppose there exists a positive function $h(r)$ on $(0,+\infty)$ such that

$$
\operatorname{Hess}(r) \geq h(r)[g-d r \otimes d r]
$$

then

$$
\sum_{i=1}^{m-1} \varepsilon_{i} \geq \begin{cases}1+(2 m-3) r h(r) & \text { if } \quad r h(r) \geq 1 \\ 2(m-1) r h(r) & \text { if } \quad r h(r)<1\end{cases}
$$

Proof. By assumptions, we have

$$
H \operatorname{Hess}\left(r^{2}\right) \geq 2 d r \otimes d r+2 r h(r)[g-d r \otimes d r]
$$

First we consider the case $r h(r) \geq 1$. Replacing $\Psi$ by $r^{2}$ in (4.9), we see that 2 $\sum_{i=1}^{m-1} \varepsilon_{i}$ is the trace of $\operatorname{Hess}\left(r^{2}\right)$ on some real $2(m-1)$ dimensional $J$-invariant subspace of $T M$. It follows from (4.15) that

$$
\sum_{i=1}^{m-1} \varepsilon_{i} \geq 1+(2 m-3) r h(r)
$$

Clearly we have

$$
\sum_{i=1}^{m-1} \varepsilon_{i} \geq 2(m-1) r h(r)
$$

if $r h(r)<1$. This proves this lemma.

Lemma 4.5. Let $(M, g)$ be a complete Riemannian manifold with a pole $x_{0}$ and $r$ the distance function relative to $x_{0}$. Denote by $K_{r}$ the radial curvature $K_{r}$ of $M$.

(i) If $K_{r} \leq 0$, then

$$
H e s s(r) \geq \frac{1}{r}[g-d r \otimes d r]
$$

(ii) If $K_{r} \leq \frac{b^{2}}{1+r^{2}}$ with $b^{2} \in[0,1 / 4]$, then

$$
\operatorname{Hess}(r) \geq \frac{1+\sqrt{1-4 b^{2}}}{2 r}[g-d r \otimes d r]
$$

(iii) If $K_{r} \leq \frac{B}{\left(1+r^{2}\right)^{1+\varepsilon}}$ with $\varepsilon>0$ and $0 \leq B<2 \varepsilon$, then

$$
\operatorname{Hess}(r) \geq \frac{1-\frac{B}{2 \varepsilon}}{r}[g-d r \otimes d r]
$$

(iv) If $K_{r} \leq-\beta^{2}$ with $\beta>0$, then

$$
H \operatorname{ess}(r) \geq \beta \operatorname{coth}(\beta r)[g-d r \otimes d r]
$$


(v) If $K_{r} \leq-\frac{a^{2}}{1+r^{2}}$ with $a>0$, then

$$
\operatorname{Hess}(r) \geq \max \left\{\frac{1+\sqrt{1+4 a^{2}}}{2(1+r)}, \frac{1}{r}\right\}[g-d r \otimes d r]
$$

Proof. The cases (i) and (iv) are standard (cf. [GW]). The case (ii) is proved in Lemma 1.2 (b) of [EF]. The case (iii) follows immediately from the quasi-isometry Theorem due to $[\mathrm{GW}]$ (cf. also [DW]). The case (v) is treated in [GW], [PRS] as an asymptotical comparison theorem. Actually we may deduce (cf. page 39 of [PRS])

$$
\operatorname{Hess}(r) \geq \frac{1+\sqrt{1+4 a^{2}}}{2(1+r)}[g-d r \otimes d r]
$$

On the other hand, the assumption $K_{r} \leq-\frac{a^{2}}{1+r^{2}}<0$ implies by (i) that

$$
\operatorname{Hess}(r) \geq \frac{1}{r}[g-d r \otimes d r]
$$

Therefore we prove $(\mathrm{v})$.

Lemma 4.6. Let $M^{m}$ be an $m$ dimensional complete Kähler manifold with a pole $x_{0}$. Let $\varepsilon_{1} \leq \varepsilon_{2} \leq \cdots \leq \varepsilon_{m}$ be the eigenvalues of $\left(\left(r^{2}\right)_{i \bar{j}}\right)$, where $r$ is the distance function relative to $x_{0}$. Suppose the radial curvature $K_{r}$ of $M$ satisfies one of the five conditions (i), (ii), (iii), (iv) and (v) in Lemma 4.5. Then

$$
\sum_{i=1}^{m-1} \varepsilon_{i} \geq \begin{cases}2 m-2 & \text { if } K_{r} \text { satisfies (i) } \\ (m-1)\left(1+\sqrt{1-4 b^{2}}\right) & \text { if } K_{r} \text { satisfies (ii) } \\ 2(m-1)\left(1-\frac{B}{2 \varepsilon}\right) & \text { if } K_{r} \text { satisfies (iii) } \\ 1+(2 m-3) \beta r \operatorname{coth}(\beta r) & \text { if } K_{r} \text { satisfies (iv) } \\ \max \left\{2 m-2,1+\frac{(2 m-3)\left(1+\sqrt{1+4 a^{2}}\right) r}{2(1+r)}\right\} & \text { if } \left.K_{r} \text { satisfies ( } v\right)\end{cases}
$$

Proof. Suppose $K_{r}$ satisfies (i). Then Lemma 4.5 and Lemma 4.4 yield immediately $\sum_{i=1}^{m-1} \varepsilon_{i} \geq 2 m-2$. This proves (i). Now we assume that $K_{r}$ satisfies (iv). Clearly $\beta r \operatorname{coth}(\beta r) \geq 1$ on $(0,+\infty)$, because the increasing function $\beta r \operatorname{coth} \beta r \rightarrow 1$ as $r \rightarrow 0$. Therefore Lemma 4.4 implies that

$$
\sum_{i=1}^{m-1} \varepsilon_{i} \geq 1+(2 m-3) \beta r \operatorname{coth}(\beta r)
$$

Hence we have proved (iv). In a similar way, we may use Lemma 4.4 and Lemma 4.5 to prove the cases (ii), (iii) and (v) too.

From Lemma 4.6, we see that if $M$ is as in Lemma 4.6, then its distance function $r$ is a special exhaustion function for $M$. From the proof of Theorem 4.3 and remark 4.2 , it follows that 
Theorem 4.7. Let $M\left(\operatorname{dim}_{C} M=m>1\right), r, K_{r}$ be as in Lemma 4.6. Suppose $f: M \rightarrow N$ is a pluriharmonic map between Kähler manifolds. Set

$$
\lambda= \begin{cases}2 m-2 & \text { if } K_{r} \text { satisfies one of (i), (iv), (v) } \\ (m-1)\left(1+\sqrt{1-4 b^{2}}\right) & \text { if } K_{r} \text { satisfies (ii) } \\ 2(m-1)\left(1-\frac{B}{2 \varepsilon}\right) & \text { if } K_{r} \text { satisfies (iii) }\end{cases}
$$

Then $f$ satisfies

$$
\frac{1}{\rho_{1}^{\lambda}} \int_{B_{\rho_{1}}\left(x_{0}\right)}|\bar{\partial} f|^{2} \leq \frac{1}{\rho_{2}^{\lambda}} \int_{B_{\rho_{2}}\left(x_{0}\right)}|\bar{\partial} f|^{2}
$$

and

$$
\frac{1}{\rho_{1}^{\lambda}} \int_{B_{\rho_{1}}\left(x_{0}\right)}|\partial f|^{2} \leq \frac{1}{\rho_{2}^{\lambda}} \int_{B_{\rho_{2}}\left(x_{0}\right)}|\partial f|^{2}
$$

for any $0<\rho_{1} \leq \rho_{2}$.

Now let $f: M \rightarrow N$ be a pluriconformal harmonic map from a Kähler manifold. By definition, $f$ satisfies

$$
<d f(J X), d f(J Y)>=<d f(X), d f(Y)>
$$

for any $X, Y \in T M$. Clearly (4.18) is equivalent to

$$
<d f(X), d f(J Y)>=-<d f(J X), d f(Y)>
$$

Theorem 4.8. Let $f: M \rightarrow N$ be a pluriconformal harmonic map between two Kähler manifolds. Suppose $M$ satisfies the conditions in Theorem 4.3 (resp. Theorem 4.7) and $\lambda$ is given by (4.4) (resp. (4.17)). Then $f$ satisfies

$$
\frac{1}{\rho_{1}^{\lambda}} \int_{B_{\Phi}\left(\rho_{1}\right)}|d f|^{2} \leq \frac{1}{\rho_{2}^{\lambda}} \int_{B_{\Phi}\left(\rho_{2}\right)}|d f|^{2} \quad\left(\text { resp. } \frac{1}{\rho_{1}^{\lambda}} \int_{B \rho_{1}}|d f|^{2} \leq \frac{1}{\rho_{2}^{\lambda}} \int_{B_{\rho_{2}}}|d f|^{2}\right)
$$

for any $0<\rho_{1} \leq \rho_{2}$.

Proof. Suppose $M$ posses a special function $\Phi$. As in the proof of Theorem 4.3, we set $X=\Phi \nabla \Phi$. Since $f$ is harmonic, it is known that $f$ satisfies a conservation law ([BE]), that is, $\operatorname{div} S_{f}=0$, where $S_{f}$ is defined by (1.6). Then (1.13) yields

$$
\int_{\partial B_{\Phi}(t)} S_{f}(X, \nu)=\int_{B_{\Phi}(t)}<S_{f}, \nabla \theta_{X}>
$$

for a regular value $t>0$ of $\Phi$. Similar to (4.7), we get

$$
S_{f}(X, \nu) \leq \frac{t \sqrt{k_{2}}}{2}|d f|^{2}
$$

By using the pluriconformality of $f$ and replacing $\sigma$ by $d f$ in (4.8), (4.11) and (4.12), we may derive the following:

$$
<S_{f}, \nabla \theta_{X}>\geq \frac{k_{1}}{2}|d f|^{2}
$$


It follows that

$$
t \sqrt{k_{2}} \int_{\partial B_{\Phi}(t)}|d f|^{2} \geq k_{1} \int_{B_{\Phi}(t)}|d f|^{2}
$$

Similar to the remaining argument in the proof of Theorem 4.3, we have

$$
\frac{1}{\rho_{1}^{\lambda}} \int_{B_{\Phi}\left(\rho_{1}\right)}|d f|^{2} \leq \frac{1}{\rho_{2}^{\lambda}} \int_{B_{\Phi}\left(\rho_{2}\right)}|d f|^{2}
$$

for any $0<\rho_{1} \leq \rho_{2}$.

Notice that the curvature tensors of the target manifolds in Theorem 4.3, Theorem 4.7 and Theorem 4.8 play no role, since we consider the conservative cases in this subsection.

\subsection{The case of harmonic maps}

Now we consider a harmonic map between Kähler manifolds. In this case, $\sigma$ and $\tau$ don't satisfy the conservation laws in general. However, if the target Kähler manifold has strongly semi-negative curvature, we will show that the integral formula (1.13) can still be used to establish the monotonicity formulae for the partial energies.

Lemma 4.9. Let $f: M \rightarrow N$ be a harmonic map from a Kähler manifold into a Kähler manifold with strongly semi-negative curvature. Let $D \subset M$ be a domain with a compact closure and non-empty smooth boundary. Let $\varphi$ be a smooth defining function for $D$ with only discrete critical point. Set $X=\nabla \varphi$. Then

$$
\int_{D}\left(\operatorname{div} S_{\sigma}\right)(X)=\int_{D}\left(\operatorname{div} S_{\tau}\right)(X) \geq 0
$$

Proof. From Lemma 1.1 and Lemma 2.1, we have

$$
\left(\operatorname{div} S_{\sigma}\right)(X)=<i_{X} d^{\nabla} \sigma, \sigma>
$$

Set $D_{t}=\{\varphi<t\}$ for $t \leq 0$. Let $\nu=\frac{\nabla \varphi}{|\nabla \varphi|}$ on $\partial D_{t}$, where $t$ is a regular value in $\operatorname{Im}(\varphi)$. By (4.20) and using the coarea formula and divergence theorem, we deduce that

$$
\begin{aligned}
\int_{D}\left(\operatorname{div} S_{\sigma}\right)(X) & =\int_{-\infty}^{0}\left(\int_{\partial D_{t}}\left(\operatorname{div} S_{\sigma}\right)\left(\frac{\nabla \varphi}{|\nabla \varphi|}\right)\right. \\
& =\int_{-\infty}^{0}\left(\int_{\partial D_{t}}<i_{\nu} d^{\nabla} \sigma, \sigma>\right) d t \\
& =\int_{-\infty}^{0}\left(\int_{\partial D_{t}} i_{\nu} \gamma\right) d t \\
& =\int_{-\infty}^{0}\left(\int_{D_{t}} \operatorname{div} \gamma\right) d t
\end{aligned}
$$

where $\gamma$ is defined by (3.20). Likewise we have

$$
\int_{D}\left(\operatorname{div} S_{\tau}\right)(X)=\int_{-\infty}^{0}\left(\int_{D_{t}} \operatorname{div} \rho\right) d t
$$


where $\rho$ is defined by (3.25). It follows from Lemma 3.2 that $\int_{D}\left(\operatorname{div} S_{\sigma}\right)(X)=$ $\int_{D}\left(\operatorname{div} S_{\tau}\right)(X) \geq 0$.

Remark 4.3.

(a) From the proof of Lemma 4.9, we see that the corresponding results also hold true if the integral domain $D$ in (4.19) is replaced by the domain $D(c, d):=\{x \in D: c<$ $\varphi<d\}$ for any $c<d \leq 0$.

(b) If $M$ is a complete Kähler manifold with a pole $x_{0}$, we may take $\varphi=\frac{1}{2} r^{2}$, where $r$ is distance function relative $x_{0}$. Set $X=r \frac{\partial}{\partial r}$ in Lemma 4.9. Then we have

$$
\int_{B_{R}}\left(\operatorname{div} S_{\sigma}\right)(X)=\int_{B_{R}}\left(\operatorname{div} S_{\tau}\right)(X) \geq 0
$$

(c) From (3.24) and(3.27), we see that if $f$ is harmonic map, but not pluriharmonic. Then $\operatorname{div}(\gamma)=\operatorname{div}(\rho)>0$ at some point of the geodesic ball $B_{R}$. Therefore $\int_{B_{R}} \operatorname{div}\left(S_{\sigma}\right)(X)=\int_{B_{R}}\left(\operatorname{div} S_{\tau}\right)(X)>0$, which implies that $\sigma$ and $\tau$ don't satisfy the conservation laws.

Theorem 4.10. Let $f: M \rightarrow N$ be a harmonic map from a complete Kähler manifold to a Kähler manifold with strongly semi-negative curvature. Suppose $M$ satisfies the conditions in Theorem 4.3 (resp. Theorem 4.7), $\lambda$ is given by (4.4) (resp. (4.17)) and set $D_{R}=B_{\Phi}(R)\left(\right.$ resp. $\left.B_{R}\left(x_{0}\right)\right)$. Then

$$
\frac{1}{\rho_{1}^{\lambda}} \int_{D_{\rho_{1}}}|\bar{\partial} f|^{2} \leq \frac{1}{\rho_{2}^{\lambda}} \int_{D_{\rho_{2}}}|\bar{\partial} f|^{2}
$$

and

$$
\frac{1}{\rho_{1}^{\lambda}} \int_{D_{\rho_{1}}}|\partial f|^{2} \leq \frac{1}{\rho_{2}^{\lambda}} \int_{D_{\rho_{2}}}|\partial f|^{2}
$$

for any $0<\rho_{1} \leq \rho_{2}$.

Proof. Without loss of generality, we assume that $M$ satisfies the conditions in Theorem 4.3. Actually we have already pointed out this result in Remark 1.1. Here we give only a brief discussion. Take $X=\nabla\left(\frac{1}{2} \Psi\right)=\Phi \nabla \Phi$. Since $N$ has strongly semi-negative curvature, we obtain by Lemma 4.9 that

$$
\int_{B_{\Phi}(R)} \operatorname{div} S_{\sigma}(X) \geq 0
$$

It follows from (1.13) and (4.24) that

$$
\int_{\partial B_{\Phi}(R)} S_{\sigma}(X, \nu) \geq \int_{B_{\Phi}(R)}<S_{\sigma}, \nabla \theta_{X}>
$$

The remaining argument is similar to that in the proof of Theorem 4.3. 


\subsection{Local monotonicity formulae}

Theorem 4.11. Let $M$ be an $m$-dimensional Kähler manifold and let $r$ denote the distance function relative to $x_{0} \in M$. Suppose the radial curvature $K_{r}$ of $M$ satisfies $K_{r} \leq K_{0}$ on $B_{R_{0}}\left(x_{0}\right)$, where $K_{0}$ is a positive constant and $R_{0}$ is a fixed positive number less than the injective radius of $M$ at $x_{0}$. Suppose $f: M \rightarrow N$ is either a pluriharmonic map into any Kähler manifold or a harmonic map into a Kähler manifold with strongly semi-negative curvature. Then

$$
\frac{e^{C\left(K_{0}\right) \rho_{2}}}{\rho_{2}^{2 m-2}} \int_{B_{\rho_{2}}\left(x_{0}\right)}|\bar{\partial} f|^{2}-\frac{e^{C\left(K_{0}\right) \rho_{1}}}{\rho_{1}^{2 m-2}} \int_{B_{\rho_{1}}\left(x_{0}\right)}|\bar{\partial} f|^{2} \geq \int_{\rho_{1}}^{\rho_{2}}\left[\frac{e^{C\left(K_{0}\right) t}}{t^{2 m-2}} \int_{\partial B_{t}\left(x_{0}\right)}\left|i_{\frac{\partial}{\partial r}} \sigma\right|^{2}\right] d t
$$

and

$$
\frac{e^{C\left(K_{0}\right) \rho_{2}}}{\rho_{2}^{2 m-2}} \int_{B_{\rho_{2}}\left(x_{0}\right)}|\partial f|^{2}-\frac{e^{C\left(K_{0}\right) \rho_{1}}}{\rho_{1}^{2 m-2}} \int_{B_{\rho_{1}}\left(x_{0}\right)}|\partial f|^{2} \geq \int_{\rho_{1}}^{\rho_{2}}\left[\frac{e^{C\left(K_{0}\right) t}}{t^{2 m-2}} \int_{\partial B_{t}\left(x_{0}\right)}\left|i_{\frac{\partial}{\partial r}} \tau\right|^{2}\right] d t
$$

for any $0<\rho_{1} \leq \rho_{2}<R_{0}$, where $C\left(K_{0}\right)$ is a constant depending on $K_{0}$.

Proof. Since $K \leq K_{0}$ on $B_{R_{0}}(p)$, we get by Hessian comparison theorem the following

$$
\operatorname{Hess}(r) \geq \sqrt{K_{0}} \cot \left(\sqrt{K_{0}} r\right)[g-d r \otimes d r]
$$

Now the strictly decreasing function $\sqrt{K_{0}} r \cot \left(\sqrt{K_{0}} r\right) \rightarrow 1$ as $r \rightarrow 0$, we have $\sqrt{K_{0}} r \cot \left(\sqrt{K_{0}} r\right)<1$ on $(0,+\infty)$. Let $\Psi=r^{2}$ and let $\varepsilon_{1} \leq \cdots \leq \varepsilon_{m}$ be the eigenvalue of $H\left(r^{2}\right)$. It follows from Lemma 4.4 that

$$
\begin{aligned}
\sum_{i=1}^{m-1} \varepsilon_{i} & \geq 2(m-1) \sqrt{K_{0}} r \cot \left(\sqrt{K_{0}} r\right) \\
& =2(m-1)+2(m-1)\left[\sqrt{K_{0}} r \cot \left(\sqrt{K_{0}} r\right)-1\right]
\end{aligned}
$$

Obviously there exists a constant positive $\widetilde{C}\left(K_{0}\right)$ such that

$$
1-\sqrt{K_{0}} r \cot \left(\sqrt{K_{0}} r\right) \leq r \widetilde{C}\left(K_{0}\right)
$$

on $B_{R_{0}}(p)$. Set $X=\frac{1}{2} \nabla \Psi$. From (4.12), (4.25) and (4.26), we get

$$
\begin{aligned}
<S_{\sigma}, \nabla \theta_{X}> & \geq\left(\sum_{j=1}^{m-1} \varepsilon_{j}\right) \frac{|\sigma|^{2}}{2} \\
& \geq\left[2(m-1)-C\left(K_{0}\right) r\right] \frac{|\sigma|^{2}}{2}
\end{aligned}
$$

where $C\left(K_{0}\right)=2(m-1) \widetilde{C}\left(K_{0}\right)$.

Notice that $|\nabla r|=1$. Then (1.8) gives

$$
S_{\sigma}(X, \nu) \leq r\left[\frac{|\sigma|^{2}}{2}-\left|i_{\nu} \sigma\right|^{2}\right]
$$


When $f$ is pluriharmonic, we know from Proposition 4.2 that $\sigma$ satisfy the conservation law. Using a similar technique as in the proof of Proposition 1.2, we can deduce from (4.27) and (4.28) the following

$$
\frac{d}{d r}\left[e^{C\left(K_{0}\right) r} r^{-(2 m-2)} \int_{B_{r}\left(x_{0}\right)}|\sigma|^{2}\right] \geq 2 r^{-(2 m-2)} e^{C\left(K_{0}\right) r} \int_{\partial B_{r}\left(x_{0}\right)}|\sigma|^{2}
$$

for $r<R_{0}$. By integration on $\left[\rho_{1}, \rho_{2}\right]$, we get the monotonicity formula for $\sigma$. Likewise we have the monotonicity formula for $\tau$.

Suppose now that $f: M \rightarrow N$ is a harmonic map into a Kähler manifold with strongly semi-negative curvature. From Lemma 4.9, Remark 4.3 and Remark 1.1, it is clear that the monotonicity formulae still hold.

Corollary 4.12. Let $B_{R_{0}}\left(x_{0}\right) \subset M, N$ and $f: M \rightarrow N$ be as in Theorem 4.11. Then

$$
\frac{e^{C(\alpha, \beta) \rho_{1}}}{\rho_{1}^{2 m-2}} \int_{B_{\rho_{1}}\left(x_{0}\right)}|\bar{\partial} f|^{2} \leq \frac{e^{C(\alpha, \beta) \rho_{2}}}{\rho_{2}^{2 m-2}} \int_{B_{\rho_{2}}\left(x_{0}\right)}|\bar{\partial} f|^{2}
$$

and

$$
\frac{e^{C(\alpha, \beta) \rho_{1}}}{\rho_{1}^{2 m-2}} \int_{B_{\rho_{1}}\left(x_{0}\right)}|\partial f|^{2} \leq \frac{e^{C(\alpha, \beta) \rho_{2}}}{\rho_{2}^{2 m-2}} \int_{B_{\rho_{2}}\left(x_{0}\right)}|\partial f|^{2}
$$

for $0<\rho_{1} \leq \rho_{2}<R_{0}$.

Next, we hope to establish the monotonicity formulae outside of a compact subset of a complete Kähler manifold.

Theorem 4.13. Let $M$ be an $m$-dimensional complete Kähler manifold. Suppose $M$ posses an exhaustion function $\Phi$ which is special outside a sublevel set $B_{\Phi}\left(R_{0}\right)$ for some $R_{0}>0$. Suppose $f: M \rightarrow N$ is either a pluriharmonic map into any Kähler manifold or a harmonic map into a Kähler manifold with strongly semi-negative curvature. Then

$$
\frac{1}{\rho_{1}^{\lambda}} \int_{B_{\Phi}\left(\rho_{1}\right)-B_{\Phi}\left(R_{0}\right)}|\bar{\partial} f|^{2} \leq \frac{1}{\rho_{2}^{\lambda}} \int_{B_{\Phi}\left(\rho_{2}\right)-B_{\Phi}\left(R_{0}\right)}|\bar{\partial} f|^{2}
$$

and

$$
\frac{1}{\rho_{1}^{\lambda}} \int_{B_{\Phi}\left(\rho_{1}\right)-B_{\Phi}\left(R_{0}\right)}|\partial f|^{2} \leq \frac{1}{\rho_{2}^{\lambda}} \int_{B_{\Phi}\left(\rho_{2}\right)-B_{\Phi}\left(R_{0}\right)}|\partial f|^{2}
$$

for any $R_{0}<\rho_{1} \leq \rho_{2}$, where $\lambda$ is defined by (4.4) on $M-B_{\Phi}\left(R_{0}\right)$.

Proof. Take $X=\frac{1}{2} \nabla \Phi^{2}=\Phi \nabla \Phi$. For any $R>R_{0}$, we set $D=B_{\Phi}(R)-B_{\Phi}\left(R_{0}\right)$. By applying the integral formula (1.13) on $D$ and arguing in a similar way as in 
Proposition 1.2, Theorem 4.3 and Theorem 4.10, we may deduce the following

$$
\begin{aligned}
& \int_{\partial B_{\Phi}(R)} S_{\sigma}(X, \nu)-\int_{\partial B_{\Phi}\left(R_{0}\right)} S_{\sigma}(X, \nu) \\
& =R<\nabla \Phi, \nu>\left\{\int_{\partial B_{\Phi}(R)} S_{\sigma}(\nu, \nu)-\int_{\partial B_{\Phi}\left(R_{0}\right)} S_{\sigma}(\nu, \nu)\right\} \\
& \geq \frac{1}{2} \int_{B_{\Phi}(R)-B_{\Phi}\left(R_{0}\right)}<S_{\sigma}, \operatorname{Hess}\left(\Phi^{2}\right)> \\
& \geq \frac{k_{1}}{2} \int_{B_{\Phi}(R)-B_{\Phi}\left(R_{0}\right)}|\sigma|^{2}
\end{aligned}
$$

By definition of $S_{\sigma}$ and (2.12), we get

$$
\begin{aligned}
S_{\sigma}(\nu, \nu) & =\frac{|\sigma|^{2}}{2}-<\sigma(\nu), \sigma(\nu)> \\
& \geq \frac{1}{2}[<\sigma(\nu), \sigma(\nu)>+<\sigma(J \nu), \sigma(J \nu)>] \\
& -<\sigma(\nu), \sigma(\nu)> \\
& =0
\end{aligned}
$$

Then (4.32) and (4.33) yield

$$
R \sqrt{k_{2}} \int_{\partial B_{\Phi}(R)} S_{\sigma}(\nu, \nu) \geq \frac{k_{1}}{2} \int_{B_{\Phi}(R)-B_{\Phi}\left(R_{0}\right)}|\sigma|^{2}
$$

It follows from the coarea formula and (4.34) that

$$
\begin{aligned}
R \frac{d}{d R}\left(\int_{B_{\Phi}(R)-B_{\Phi}\left(R_{0}\right)}|\sigma|^{2}\right) & \geq \frac{R}{\sqrt{k_{2}}} \int_{\partial B_{\Phi}(R)}|\sigma|^{2} \\
& \geq \frac{2 R}{\sqrt{k_{2}}} \int_{\partial B_{\Phi}(R)} S_{\sigma}(\nu, \nu) \\
& \geq \lambda \int_{B_{\Phi}(R)-B_{\Phi}\left(R_{0}\right)}|\sigma|^{2}
\end{aligned}
$$

which implies that

$$
\frac{d}{d r}\left\{R^{-\lambda} \int_{B_{\Phi}(R)-B_{\Phi}\left(R_{0}\right)}|\bar{\partial} f|^{2}\right\} \geq 0
$$

By integrating (4.35) on $\left[\rho_{1}, \rho_{2}\right]$, we get (4.30). Likewise we have (4.31).

As an application of Theorem 4.20, we give the following 
Theorem 4.14. Let $M$ be a complete Kähler manifold with a pole $x_{0}$. Suppose the radial curvature $K_{r}$ of $M$ satisfies one of the following two conditions

(a) $K_{r} \leq-\beta^{2}$ with $\beta>0$;

(b) $K_{r} \leq-\frac{a^{2}}{1+r^{2}}$ with $a>0$.

Suppose $f: M \rightarrow N$ is either a pluriharmonic map into a Kähler manifold or a harmonic map into a Kähler manifold with strongly semi-negative curvature. Set

$$
\lambda_{0}= \begin{cases}1+(2 m-3) \beta R_{0} \operatorname{coth}\left(\beta R_{0}\right) & \text { if } K_{r} \text { satisfies (a) } \\ C\left(R_{0}\right) & \text { if } K_{r} \text { satisfies }(b)\end{cases}
$$

for any $R_{0}>0$, where

$$
C\left(R_{0}\right)= \begin{cases}1+\frac{(2 m-3)\left(1+\sqrt{1+4 a^{2}}\right) R_{0}}{2\left(1+R_{0}\right)} & \text { if } \frac{\left(1+\sqrt{1+4 a^{2}}\right) R_{0}}{2\left(1+R_{0}\right)} \geq 1 \\ \frac{(m-1)\left(1+\sqrt{1+4 a^{2}}\right) R_{0}}{\left(1+R_{0}\right)} & \text { if } \frac{\left(1+\sqrt{1+4 a^{2}}\right) R_{0}}{2\left(1+R_{0}\right)}<1\end{cases}
$$

Then

$$
\frac{1}{\rho_{1}^{\lambda_{0}}} \int_{B_{\rho_{1}}\left(x_{0}\right)-B_{R_{0}}\left(x_{0}\right)}|\bar{\partial} f|^{2} \leq \frac{1}{\rho_{2}^{\lambda_{0}}} \int_{B \rho_{2}\left(x_{0}\right)-B_{R_{0}}\left(x_{0}\right)}|\bar{\partial} f|^{2}
$$

and

$$
\frac{1}{\rho_{1}^{\lambda_{0}}} \int_{B_{\rho_{1}}\left(x_{0}\right)-B_{R_{0}}\left(x_{0}\right)}|\partial f|^{2} \leq \frac{1}{\rho_{2}^{\lambda_{0}}} \int_{B \rho_{2}\left(x_{0}\right)-B_{R_{0}}\left(x_{0}\right)}|\partial f|^{2}
$$

for any $R_{0}<\rho_{1} \leq \rho_{2}$.

Proof. For the case $K_{r} \leq-\beta^{2}$, we have from Lemma 4.4, Lemma 4.5 and the proof of Lemma 4.6, we have $\sum_{i=1}^{m-1} \varepsilon_{i}(x) \geq 1+(2 m-3) \beta R_{0} \operatorname{coth}\left(\beta R_{0}\right)$ for $x \in M-B_{R_{0}}\left(x_{0}\right)$. Then Theorem 4.13 follows immediately from Theorem 4.14. In a similar way, we may prove the case (b) by Lemma 4.4 and Lemma 4.5 and using the fact that the function $\frac{r}{1+r}$ is increasing.

Notice that (4.33) is the key property that allows us to establish the monotonicity formulae outside a sublevel set of the exhaustion function $\Phi$ in Theorem 4.13. Suppose now that $f: M \rightarrow N$ is a pluriconformal harmonic map from a Kähler manifold. We may consider the stress energy tensor $S_{f}$ as in Theorem 4.8. Let $\nu$ be the unit outward normal vector field of $B_{\Phi}\left(R_{0}\right)$. By (4.18), we also have

$$
S_{f}(\nu, \nu) \geq 0
$$

Similar to the arguments in Theorem 4.13 (see also Theorem 4.8), it is easy to deduce from (4.38) the following:

Theorem 4.15. Let $M, \Phi, R_{0}$ and $\lambda$ be as in Theorem 4.13. Suppose $f: M \rightarrow N$ is a pluriconformal harmonic map. Then

$$
\frac{1}{\rho_{1}^{\lambda}} \int_{B_{\Phi}\left(\rho_{1}\right)-B_{\Phi}\left(R_{0}\right)}|d f|^{2} \leq \frac{1}{\rho_{2}^{\lambda}} \int_{B_{\Phi}\left(\rho_{2}\right)-B_{\Phi}\left(R_{0}\right)}|d f|^{2}
$$

for any $R_{0}<\rho_{1} \leq \rho_{2}$. 
Corollary 4.16. Let $M, K_{r}, R_{0}$ and $\lambda_{0}$ be as in Theorem 4.14. Suppose $f: M \rightarrow N$ is a pluriconformal harmonic map. Then

$$
\frac{1}{\rho_{1}^{\lambda_{0}}} \int_{B_{\rho_{1}}-B_{R_{0}}}|d f|^{2} \leq \frac{1}{\rho_{2}^{\lambda_{0}}} \int_{B_{\rho_{2}}-B_{R_{0}}}|d f|^{2}
$$

for any $R_{0}<\rho_{1} \leq \rho_{2}$.

\section{Holomorphicity and constancy of harmonic maps}

In this section, we derive some results about holomorphicity and constancy of harmonic maps between Kähler manifolds. Most of these results are direct consequences of the monotonicity formulae in last section.

Theorem 5.1. Let $M, \Phi, R_{0}$ and $\lambda$ be as in Theorem 4.13, that is, $\Phi$ is an exhaustion function on $M$, which is special on $M-B_{\Phi}\left(R_{0}\right)$. Suppose $f: M \rightarrow N$ is either a pluriharmonic map into a Kähler manifold or a harmonic map into a Kähler manifold with strongly semi-negative curvature. If

$$
\int_{B_{\Phi}(R)}|\bar{\partial} f|^{2}=o\left(R^{\lambda}\right) \quad\left(\text { resp. } \quad \int_{B_{\Phi}(R)}|\partial f|^{2}=o\left(R^{\lambda}\right)\right) \quad \text { as } R \rightarrow \infty
$$

then $f$ is holomorphic (resp. anti-holomorphic). In particular, if $E^{\prime \prime}(f)<+\infty$ (resp. $\left.E^{\prime}(f)<+\infty\right)$, then $f$ is holomorphic (resp. anti-holomorphic).

Proof. The assumption $\int_{B_{\Phi}(R)}|\bar{\partial} f|^{2}=o\left(R^{\lambda}\right)\left(\right.$ resp. $\left.\int_{B_{\Phi}(R)}|\partial f|^{2}=o\left(R^{\lambda}\right)\right)$ as $R \rightarrow \infty$ is equivalent to

$$
\int_{B_{\Phi}(R)-B_{\Phi}\left(R_{0}\right)}|\bar{\partial} f|^{2}=o\left(R^{\lambda}\right) \quad\left(\operatorname{resp} . \int_{B_{\Phi}(R)}|\partial f|^{2}=o\left(R^{\lambda}\right)\right) \text { as } R \rightarrow \infty
$$

By (4.30) (resp.(4.31)), we deduce that $f$ is holomorphic (resp. anti-holomorphic) on $M-B_{\Phi}\left(R_{0}\right)$. Since $f$ is harmonic, it then follows as in [Si1] from the unique continuation property that $\bar{\partial} f=0$ (resp. $\partial f=0$ ) on the whole $M$. This proves the theorem.

Corollary 5.2. Let $M, \Phi, \lambda$ be as in Theorem 4.3. Suppose $f: M \rightarrow N$ is either a pluriharmonic map into a Kähler manifold or a harmonic map into a Kähler manifold with strongly semi-negative curvature. If $f$ satisfies the growth condition as in Theorem 5.1, then $f$ is holomorphic (resp. anti-holomorphic).

Corollary 5.3. Let $M, \Phi, \lambda, f$ and $N$ be as in Corollary 5.2. If $f$ satisfies

$$
\int_{B_{\Phi}(R)}|d f|^{2}=o\left(R^{\lambda}\right) \quad \text { as } \quad R \rightarrow \infty
$$

then $f$ is constant. In particular, if $E(f)<+\infty$, then $f$ is constant.

Proof. By Corollary 5.2, $f$ is both holomorphic and anti-holomorphic. Hence $f$ must be constant.

According to $[\mathrm{Wu}]$, a function on a manifold is called quasipositive if it is everywhere nonnegative and is positive at one point. 
Proposition 5.4. Let $M$ be a Kähler manifold possing a function $\Phi$ with the properties (4.1), (4.2) and that $\sum_{i=1}^{m-1} \varepsilon_{i}$ is quasipositive, where $\varepsilon_{1} \leq \varepsilon_{2} \leq \cdots \leq \varepsilon_{m}$ are the eigenvalues of the complex Hessian $H\left(\Phi^{2}\right)$. Suppose $f: M \rightarrow N$ is either a pluriharmonic map into a Kähler manifold or a harmonic map into a Kähler manifold with strongly semi-negative curvature. If

$$
\int_{M}|\bar{\partial} f|^{2}<\infty \quad\left(\text { resp. } \int_{M}|\partial f|^{2}<\infty\right)
$$

then $f$ is holomorphic (resp. anti-holomorphic). In particular, if $\int_{M}|d f|^{2}<\infty$ then $f$ is constant.

Proof. First we assume that $f$ is a pluriharmonic map satisfying $E^{\prime \prime}(f)<\infty$. Set $X=\frac{1}{2} \nabla\left(\Phi^{2}\right)$. Similar arguments as in the proof of Theorem 4.3 yield

$$
<S_{\sigma}, \nabla \theta_{X}>\geq\left(\sum_{i=1}^{m-1} \varepsilon_{i}\right)|\bar{\partial} f|^{2}
$$

From Proposition 4.2, (1.12) and (5.4), we obtain

$$
\operatorname{div}\left(i_{X} S_{\sigma}\right) \geq\left(\sum_{i=1}^{m-1} \varepsilon_{i}\right)|\bar{\partial} f|^{2}
$$

Clearly $\left|i_{X} S_{\sigma}\right| \leq C\left(k_{2}\right) \Phi|\bar{\partial} f|^{2}$ for some constant $C\left(k_{2}\right)$. Thus

$$
\frac{1}{R} \int_{B_{\Phi}(2 R)-B_{\Phi}(R)}\left|i_{X} S_{\sigma}\right| \leq 2 C\left(k_{2}\right) \int_{B_{\Phi}(2 R)-B_{\Phi}(R)}|\bar{\partial} f|^{2}
$$

By $E^{\prime \prime}(f)<\infty$, we get

$$
\liminf _{R \rightarrow \infty} \frac{1}{R} \int_{B_{\Phi}(2 R)-B_{\Phi}(R)}\left|i_{X} S_{\sigma}\right|=0
$$

Then Lemma 3.3 yields

$$
\int_{M} \operatorname{div}\left(i_{X} S_{\sigma}\right)=0
$$

It follows from (5.5) and (5.6) that

$$
\int_{M}\left(\sum_{i=1}^{m-1} \varepsilon_{i}\right)|\bar{\partial} f|^{2}=0
$$

Since $\sum_{i=1}^{m-1} \varepsilon_{i}$ is quasipositive, $|\bar{\partial} f|^{2}$ vanishes on an open subset of $M$. Consequently $\bar{\partial} f=0$ on the whole $M$, that is, $f$ is holomorphic.

Suppose now that $f: M \rightarrow N$ is a harmonic map into a Kähler manifold. If $E^{\prime \prime}(f)<\infty$, then Corollary 3.7 implies that $f$ is pluriharmonic. The remaining arguments are obvious. Thus we have proved this proposition. 
Theorem 5.5. Let $M$ be a complete Kähler manifold with a pole $x_{0}$. Suppose the radial curvature $K_{r}$ of $M$ satisfies one of the following three conditions:

(i) $K_{r} \leq 0$;

(ii) $K_{r} \leq \frac{b^{2}}{1+r^{2}}$ with $b \in(0,1 / 4]$;

(iii) $K_{r} \leq \frac{B}{\left(1+r^{2}\right)^{1+\varepsilon}}$ with $0<B<2 \varepsilon$.

Suppose $f: M \rightarrow N$ is either a pluriharmonic map into a Kähler manifold or a harmonic map into a Kähler manifold with strongly semi-negative curvature. If

$$
\int_{B_{r}}|\bar{\partial} f|^{2}=o\left(r^{\lambda}\right) \quad\left(\text { resp. } \quad \int_{B_{r}}|\partial f|^{2}=o\left(r^{\lambda}\right)\right) \quad \text { as } r \rightarrow \infty
$$

where

$$
\lambda= \begin{cases}2 m-2 & \text { if } K_{r} \text { satisfies (i) } \\ (m-1)(1+\sqrt{1-4 b)} & \text { if } K_{r} \text { satisfies (ii) } \\ 2(m-1)\left(1-\frac{B}{2 \varepsilon}\right) & \text { if } K_{r} \text { satisfies (iii) }\end{cases}
$$

then $f$ is holomorphic (resp. anti-holomorphic). In particular, if $E^{\prime \prime}(f)<+\infty$ (resp. $\left.E^{\prime}(f)<+\infty\right)$, then $f$ is holomorphic (resp. anti-holomorphic).

Proof. This follows directly from Theorem 4.7 and Theorem 4.10.

Remark 5.1. The author in [Wa] proved that any harmonic map $f: C^{m} \rightarrow C^{n}$ with $E^{\prime \prime}(f)<+\infty$ is holomorphic.

Theorem 5.6. Let $M$ be a complete Kähler manifold whose radial curvature $K_{r}$ satisfies one of the following two conditions

(iv) $K_{r} \leq-\beta^{2}$ with $\beta>0$;

(v) $K_{r} \leq-\frac{a^{2}}{1+r^{2}}$ with $a>0$.

Suppose $f: M \rightarrow N$ is either a pluriharmonic map into a Kähler manifold or a harmonic map into a Kähler manifold with strongly semi-negative curvature. If

$$
\int_{B_{r}\left(x_{0}\right)}|\bar{\partial} f|^{2}=o\left(r^{\Lambda_{0}}\right) \quad \text { as } r \rightarrow \infty
$$

where $\Lambda_{0}$ is either any positive number if $K_{r}$ satisfies (iv) or any positive number less than $1+\frac{(2 m-3)\left(1+\sqrt{1+4 a^{2}}\right)}{2}$ if $K_{r}$ satisfies $(v)$, then $f$ is holomorphic. In particular, if $E^{\prime \prime}(f)<+\infty$, then $f$ is holomorphic. Likewise, if we replace $\bar{\partial} f$ by $\partial f$ in the previous conditions, then $f$ is anti-holomorphic.

Proof. First we assume that $K_{r}$ satisfies (iv) and $f$ satisfies (5.9). Since $x \operatorname{coth} x$ is a nondecreasing function for $x \geq 0$ and $\lim _{x \rightarrow+\infty} \operatorname{coth} x=1$, there exists an $R_{0}$ such that $1+(2 m-3) \beta R_{0} \operatorname{coth}\left(\beta R_{0}\right) \geq \Lambda_{0}$. Then Theorem 4.14 implies that $f$ is holomorphic.

Next we assume that $K_{r}$ satisfies (v) and $f$ satisfies (5.9) too. Notice that $\frac{r}{1+r}$ is an increasing function for $r \geq 0$ and $\lim _{r \rightarrow \infty} \frac{r}{1+r}=1$. Clearly $\frac{\left(1+\sqrt{1+4 a^{2}}\right) R_{0}}{2\left(1+R_{0}\right)}>1$ and 
$1+\frac{(2 m-3)\left(1+\sqrt{1+4 a^{2}}\right) R_{0}}{2\left(1+R_{0}\right)} \geq \Lambda_{0}$ for a sufficiently large $R_{0}$. Hence we get from Theorem 4.14 that $f$ is holomorphic.

Remark 5.2. Let $f: M \rightarrow N$ be a harmonic map from any complete Kähler manifold into a Kähler manifold with strongly semi-negative curvature. Notice that we assert in Corollary 3.7 (see also Remark 3.2), without assuming any curvature conditions on $M$, that if one of the partial energy has growth order less than or equal to 2 then $f$ is pluriharmonic. Suppose now that the radial curvature $K_{r}(M)$ satisfies one of the conditions in Theorem 4.7. We assert above that if the partial energy has growth order less than $\lambda$, then $f$ is \pm holomorphic. In most cases, $\lambda$ given by (4.17) may be larger than 2 . However, it is easy to verify that $\lambda$ is less than or equal to 2 in following cases:

$$
\begin{cases}m=2 & \text { if } K_{r} \text { satisfies one of (i), (ii) } \\ 2 \leq m \leq 1+\frac{1}{1-\frac{B}{2 \varepsilon}} & \text { if } K_{r} \text { satisfies (iii) }\end{cases}
$$

Remarkably we may conclude for the case (iv) of Theorem 5.6 that if the $E^{\prime \prime}$-energy (resp. $E^{\prime}$-energy) has polynomial growth in $r$, then $f$ is holomorphic (resp. antiholomorphic).

Corollary 5.7. Let $M, N, f, \lambda$ and $\Lambda_{0}$ be as in Theorem 5.5 and Theorem 5.6. Suppose $f: M \rightarrow N$ is either a pluriharmonic map into a Kähller manifold or a harmonic map into a Kähler manifold with strongly semi-negative curvature. If $f$ satisfies

$$
\int_{B_{r}}|d f|^{2}= \begin{cases}o\left(r^{\lambda}\right) & \text { if } M \text { is as in Theorem5.5 } \\ o\left(r^{\Lambda_{0}}\right) & \text { if } M \text { is as in Theorem5.6 }\end{cases}
$$

Then $f$ is constant. In particular, if $E(f)<+\infty$, then $f$ is constant.

Proof. It follows immediately from Theorem 5.5 and Theorem 5.6 that $f$ is both holomorphic and anti-holomorphic. Hence $f$ must be constant.

Theorem 5.8. Let $M$ be as in Theorem 4.15 (resp. Corollary 4.16). Suppose $f$ : $M \rightarrow N$ is a pluriconformal harmonic map from the Kähler manifold $M$. If

$$
\int_{B_{\Phi}(R)}|d f|^{2}=o\left(R^{\lambda}\right) \quad\left(\text { resp. } \int_{B_{r}}|d f|^{2}=o\left(r^{\lambda_{0}}\right)\right)
$$

then $f$ is constant. In particular, if $E(f)<+\infty$, then $f$ is constant.

Proof. This theorem follows immediately from Theorem 4.15 (resp. Corollary 4.16) and the unique continuation theorem of harmonic maps (cf. [EL]).

Remark 5.3.

(a) For most of the results in this paper, we have to assumed $m>1$ for the conditions on $M$. We cannot expect in general that the corresponding results hold in the case of $m=1$. For example, there exit many harmonic maps from $S^{2} \rightarrow C P^{N}$ which are neither holomorphic nor anti-holomorphic (cf. [EW]). Since $R^{2}$ is conformally equivalent to $S^{2} \backslash\{p\}$, it follows that there exit many harmonic maps $R^{2} \rightarrow C P^{N}$ of 
finite energy, which are neither holomorphic nor anti-holomorphic.

(b) To establish Liouville Theorems for a harmonic map $f$, one may also consider the stress-energy tensor $S_{f}$. Usually some curvature pinching conditions on the domain manifolds are needed to obtain the Liouville theorems under the energy growth conditions (cf. [DW] for details). Note that in Corollary 5.7 or Theorem 5.8, we only assume some suitable upper bounds for $K_{r}$ to establish the Liouville theorems.

In Proposition 3.4, Corollary 3.5, Theorem 3.6 and Corollary 3.7, we have deduced the pluriharmonicity of harmonic maps from any complete Kähler manifold $M$ to a Kähler manifold with strongly semi-negative curvature under some mild growth conditions about the harmonic maps. In particular, all these harmonic maps satisfy (3.34). When $N$ is an irreducible Hermitian symmetric space, we define an integer $P(N)$ as follows (cf. [Si2]):

$$
P(N)= \begin{cases}(p-1)(q-1)+1 & \text { if } \widetilde{N}=D^{I p q} \\ \frac{1}{2}(p-2)(p-3)+1 & \text { if } \widetilde{N}=D^{I I p} \\ \frac{1}{2} p(p-1)+1 & \text { if } \widetilde{N}=D^{I I p} \\ 2 & \text { if } \widetilde{N}=D^{I V p} \\ 6 & \text { if } \widetilde{N}=D^{V} \\ 11 & \text { if } \widetilde{N}=D^{V I}\end{cases}
$$

where $\widetilde{N}$ denotes the universal covering space of $N$. We have the following:

Lemma 5.9. (cf. [Si1,2]) Let $f: M \rightarrow N$ be a smooth map between two Kähler manifolds. Then $f$ is holomorphic or anti-holomorphic, provided (i) $N$ has strongly negative curvature tensor, $f$ is a harmonic map with (3.34) and $\max _{M} r a n k_{R} d f \geq 4$, or (ii) $N$ is an irreducible Hermitian symmetric space of noncompact type, $f$ is a pluriharmonic map with $\max _{M}$ rank $_{R} d f \geq 2 P(N)+1$, where $P(N)$ is given by (5.11).

From Lemma 5.9, we obtain that:

Theorem 5.10. Let $f: M \rightarrow N$ be a harmonic map from a complete Kähler manifold into a Kähler manifold. Suppose $M$ and $f$ satisfy the conditions as in one of Proposition 3.4, Corollary 3.5, Theorem 3.6 and Corollary 3.7. Then $f$ is holomorphic or anti-holomorphic, provided that (i) $N$ has strongly negative curvature tensor and $\max _{M} \operatorname{rank}_{R} d f \geq 4$, or (ii) $N$ is an irreducible Hermitian symmetric space of noncompact type and $\max _{M}$ rank $_{R} d f \geq 2 P(N)+1$.

\section{Harmonic maps with CR Dirichlet boundary-values}

Let $D \subset M^{m}$ be a relatively compact domain with smooth connected boundary $\partial D$. For $p \in \partial D$, we denote by $H_{p}(\partial D)$ the real $2 m-2$ dimensional subspace of $T_{p}(\partial D)$ which is $J$ invariant. The distribution $H:=\left\{H_{p}: p \in \partial D\right\}$ on $\partial D$ is called the holomorphic distribution of $\partial D$. Suppose $f: \partial D \rightarrow N$ is a map into a Kähler manifold $N$. We say that $f$ satisfies the tangential Cauchy-Riemann equation $\bar{\partial}_{b} f=0$ on $\partial D$ if for every point $p \in \partial D, \Pi^{1,0} \circ d f(\xi)=0$ for any $\xi \in H_{p}^{C} \cap T_{p}^{0,1} M$, where $\Pi^{1,0}: T N \otimes C \rightarrow T^{1,0} N$ is the natural projection. It is easy to verify that $f$ satisfies 
the tangential Cauchy-Riemann equation if and only if $\sigma$ annihilates any tangent vector in the holomorphic distribution $H$.

Theorem 6.1. Let $\bar{D}_{i} \subset M(i=1,2)$ be two connected, compact smooth domains in a Kähler manifold such that $D_{1} \subset \subset D_{2}$. Set $D=D_{2}-\bar{D}_{1}$. Suppose there exists a function $\Psi \in C^{2}(\bar{D})$ satisfying the following properties:

(i) $k_{1}(x)=\sum_{i=1}^{m-1} \varepsilon_{i}(x)$ is quasipositive on $D$, where $\varepsilon_{1} \leq \varepsilon_{2} \leq \cdots \leq \varepsilon_{m}$ are the eigenvalues of the complex Hessian $\left(\Psi_{i \bar{j}}\right)$;

(ii) $\nabla \Psi=w(x) \nu$ on $\partial D_{1}$ with $w(x) \geq 0$ for every $x \in \partial D_{1}$, where $\nu$ is the unit outward normal vector field along $\partial D_{1}$.

Suppose $f: D \rightarrow N$ is a pluriharmonic map into a Kähler manifold $N$ such that $f \in C^{2}(\bar{D}, N)$ and $\bar{\partial}_{b} f=0$ on $\partial D_{2}$. Then $f$ is holomorphic.

Proof. Take $X=\frac{1}{2} \nabla \Psi$. Since $f: \bar{D} \rightarrow N$ is a pluriharmonic map, it follows from Proposition 4.2 that $f$ satisfies the conservation law. By (1.14), we get

$$
\int_{\partial D_{2}} S_{\sigma}(X, \nu)-\int_{\partial D_{1}} S_{\sigma}(X, \nu)=\int_{D}<S_{\sigma}, \nabla \theta_{X}>
$$

Similar to (4.12), we deduce that

$$
\begin{aligned}
<S_{\sigma}, \nabla \theta_{X}> & =\frac{1}{2}<S_{\sigma}, \operatorname{Hess}(\Psi)> \\
& \geq k_{1}(x)|\bar{\partial} f|^{2}
\end{aligned}
$$

on $D$. For any $x \in \partial D_{2}$, we have

$$
\begin{aligned}
2 S_{\sigma}(X, \nu) & =\frac{|\sigma|_{x}^{2}}{2}<\nabla \Psi, \nu>_{x}-<\sigma(\nabla \Psi), \sigma(\nu)>_{x} \\
& =\frac{|\sigma|_{x}^{2}}{2}<\nabla \Psi, \nu>_{x}-<\nabla \Psi, \nu>_{x}|\sigma(v)|_{x}^{2}-<\sigma\left((\nabla \Psi)_{T}\right), \sigma(\nu)>_{x}
\end{aligned}
$$

where $(\nabla \Psi)_{T}$ denotes the tangential projection of $\nabla \Psi$ on $T(\partial D)$. Since $\bar{\partial}_{b} f=0$ on $\partial D_{2}$, we have

$$
\sigma\left((\nabla \Psi)_{T}\right)=<\nabla \Psi, J \nu>\sigma(J \nu)
$$

From (2.12), we derive that

$$
|\sigma(\nu)|^{2}=|\sigma(J \nu)|^{2}
$$

and

$$
\begin{aligned}
<\sigma(J \nu), \sigma(\nu)> & =<\sigma\left(J^{2} \nu\right), \sigma(J \nu)> \\
& =-<\sigma(\nu), \sigma(J \nu)>
\end{aligned}
$$

that is,

$$
<\sigma(J \nu), \sigma(\nu)>=0
$$


Therefore

$$
\begin{aligned}
2 S_{\sigma}(X, \nu) & =<\nabla \Psi, \nu>\left[\frac{|\sigma|_{q}^{2}}{2}-|\sigma(\nu)|^{2}\right] \\
& =<\nabla \Psi, \nu>\left[\frac{|\sigma(\nu)|^{2}+|\sigma(J \nu)|^{2}}{2}-|\sigma(\nu)|^{2}\right] \\
& =0
\end{aligned}
$$

on $\partial D_{2}$. Similar to the argument in (4.33), we have the following

$$
\begin{aligned}
S_{\sigma}(X, \nu) & =\frac{w}{2} S_{\sigma}(\nu, \nu) \\
& \geq 0
\end{aligned}
$$

on $\partial D_{1}$. From $(6.1),(6.2),(6.6)$ and $(6.7)$, we have

$$
\int_{D} k_{1}(x)|\bar{\partial} f|^{2} \leq 0
$$

By assumption $k_{1}(x)>0$ at some point $x \in D$, then (6.8) implies that $\bar{\partial} f \equiv 0$ in a neighborhood $U$ of $p$. It follows that $f$ is holomorphic on $D$.

It is clear that if $\bar{D}_{1}$ is a sublevel set of the function $\Psi$, then the condition (ii) in Theorem 6.1 is automatically satisfied.

Corollary 6.2. Let $M, D_{i}(i=1,2)$ and $f$ be as in Theorem 6.1. Suppose there exists a function $\Psi \in C^{2}(\bar{D})$ satisfying the condition (i) of Theorem 6.1. Suppose there is a real number $c$ such that $D_{1} \subset\{q \in D: \Psi(q) \leq c\} \subset \subset D$. Then $f$ is holomorphic on $D$.

Proof. Set $\widehat{D}_{1}=\{q \in D: \Psi(q)<c\}$ and $\widehat{D}=D_{2}-\widehat{D}_{1}$. Applying Theorem 6.1 to the pluriharmonic map $f: \widehat{D} \rightarrow N$, we deduce that $f$ is holomorphic on $\widehat{D}$. Hence $f$ is holomorphic on $D$.

Note also that if $D_{1}=\emptyset$, the condition (ii) of Theorem 6.1 is void. Therefore we get

Corollary 6.3. Let $\bar{D} \subset M$ be a compact domain in a Kähler manifold with smooth connected boundary. Suppose there exists a function $\Psi \in C^{2}(\bar{D})$ satisfying the property that the function $k_{1}(x)=\sum_{i=1}^{m-1} \varepsilon_{i}(x)$ is quasipositive on $D$, where $\varepsilon_{1} \leq \varepsilon_{2} \leq$ $\cdots \leq \varepsilon_{m}$ are the eigenvalues of the complex Hessian $\left(\Psi_{i \bar{j}}\right)$. Suppose $f: D \rightarrow N$ is a pluriharmonic map into a Kähler manifold $N$ such that $f \in C^{2}(\bar{D}, N)$ and $\bar{\partial}_{b} f=0$ on $\partial D$. Then $f$ is holomorphic.

Corollary 6.4. Let $M$ be as in Lemma 4.6 and let $\bar{D} \subset M$ be any compact domain in $M$ with smooth connected boundary. Suppose $f: D \rightarrow N$ is a pluriharmonic map into a Kähler manifold $N$ such that $f \in C^{2}(\bar{D}, N)$ and $\bar{\partial}_{b} f=0$ on $\partial D$. Then $f$ is holomorphic. 
Proof. Take $\Psi=r^{2}$. Then Lemma 4.6 implies that $\Psi$ satisfies the assumptions of Corollary 6.3 on any connected, compact smooth domain $D$. Hence we prove this corollary.

Remark 6.1. From the proof of Theorem 6.1, it is easy to deduce the following result. Suppose $f: D \rightarrow N$ is a pluriconformal harmonic map from $D$. If $\left.d f\right|_{H}=0$ for the holomorphic distribution $H$ of $\partial D_{2}$, then $f$ is constant. Here we have to use the unique continuation theorem of harmonic maps. Hence we have the corresponding results of Corollary 6.2, Corollary 6.3 and Corollary 6.4 for pluriconformal harmonic maps. However, the conclusion for $f$ is the constancy instead of the holomorphicity.

Notice that we don't assume any convexity conditions about $\partial D$ and any curvature conditions on the target manifold in Theorem 6.1, Corollary 6.2, Corollary 6.3 and Corollary 6.4. In addition, the conditions about $\Psi$ in Corollary 6.3 are weaker than those required in Theorem 4.3.

Let $M^{m}$ be an $m$ dimensional Kähler manifold and let $q$ be a positive integer less than or equal to $m$. A function $\varphi: M \rightarrow R$ of class $C^{2}$ is said to be $q$ - plurisubharmonic (resp. strictly $q$-plurisubharmonic) if, for each point $x \in M$, the trace of the restriction of the complex Hessian $H(\varphi)$ to any $q$ dimensional complex vector subspace of $T_{x} M$ is nonnegative (resp. positive).

Let $D$ be a relatively compact domain in a Kähler manifold $M^{m}$ with smooth boundary $\partial D$. Recall that if $\varrho$ is a defining function for $D$, the Levi-form $L(\varrho)$ of $\varrho$ at $x \in \partial D$ is defined as the restriction of the complex Hessian $H_{x}(\varrho)$ to the complex subspace $H_{x}^{1,0}(\partial D)$ of $T_{x}^{C}(\partial D)$. According to [Si2], we say that $D$ or $\partial D$ is hyper- $q$-convex (resp. strongly hyper- $q$-convex) if $D$ has a smooth defining function $\varrho$ such that the eigenvalues $\lambda_{1}, \ldots, \lambda_{m-1}$ of the Levi-form $L(\varrho)$ on the holomorphic distribution $H_{x}(\partial D)$ at each point $x \in \partial D$ satisfy $\sum_{i=1}^{q} \lambda_{j_{i}} \geq 0$ (resp. $>0$ ) for all $1 \leq j_{i} \leq m-1$. The following Lemma is known (cf. [Si2], [NS]):

Lemma 6.5. Let $D$ be a hyper- $(m-1)$-convex domain in a Kähler manifold $M^{m}$ with smooth boundary. Let $f: D \rightarrow N$ be a harmonic map into a Kähler manifold with strongly seminegative curvature such that $f \in C^{2}(\bar{D}, N)$ and $\bar{\partial}_{b} f=0$ on $\partial D$. Then $f$ is pluriharmonic.

We now give an alternative proof of the following result in [CL].

Proposition 6.6. ([CL]) Let $M^{m}$ be a Kähler manifold and D a hyper- $(m-1)$ convex domain in $M$ with smooth boundary. Suppose there exists a plurisubharmonic function $\Psi \in C^{2}(\bar{D})$ so that $\left(\Psi_{i \bar{j}}\right)$ has at least two positive eigenvalues at some point in D. Let $u: \bar{D} \rightarrow N$ be a smooth map into a complete Kähler manifold with strongly seminegative curvature. If $u$ satisfies the tangential Cauchy-Riemann equation $\bar{\partial}_{b} u=$ 0 on $\partial D$, then there exists a unique holomorphic extension $f$ of $u$.

Proof. Clearly the strongly semi-negativity of the curvature tensor of $N$ implies the nonpositivity of the sectional curvature of $N$. By the existence theorem of Hamilton [Ha] and Schoen [Sc], there exists a unique harmonic map $f: D \rightarrow N$ smooth up to boundary which solves the Dirichlet problem $f=u$ on $\partial D$.

By Lemma 6.5, we know that $f$ is pluriharmonic. Clearly $\Psi$ satisfies the conditions of Corollary 6.3. Hence we conclude that $f$ is holomorphic. 
Remark 6.2. The notions of hyperconvex domains in [CL] is equivalent to the notion of hyper- $(m-1)$-convex domains defined here.

Theorem 6.7. Let $\bar{D} \subset M$ be a compact connected domain in a Kähler manifold with a smooth defining function $\Psi$. Set $D(c, 0)=\{x \in D: c<\Psi(x)<0\}$ for some $c<0$. Suppose the function $\Psi$ is $(m-1)$-plurisubharmonic on $D(c, 0)$ and strictly $(m-1)$-plurisubharmonic at some point of $D(c, 0)$. Suppose $N$ is a complete Kähler manifold with strongly seminegative curvature. Let $u: \bar{D} \rightarrow N$ be a smooth map satisfying the tangential Cauchy-Riemann equation $\bar{\partial}_{b} u=0$ on $\partial D$. Then there exists a unique holomorphic extension of $u$.

Proof. As in the proof of Proposition 6.6, we have a unique harmonic map $f: D \rightarrow N$ smooth up to boundary which solves the Dirichlet problem $f=u$ on $\partial D$.

Let $\varepsilon_{1} \leq \varepsilon_{2} \leq \cdots \leq \varepsilon_{m}$ be the eigenvalues of the complex Hessian $H(\Psi)$. Set $X=\frac{1}{2} \nabla \Psi$ and $k_{1}(x)=\sum_{i=1}^{m-1} \varepsilon_{i}(x)$ for $x \in D$. From (1.13), Lemma 4.9 and Remark 4.3, we have

$$
\int_{\partial D} S_{\sigma}(X, \nu)-\int_{\partial D_{c}} S_{\sigma}(X, \nu) \geq \int_{D(c, 0)}<S_{\sigma}, \nabla \theta_{X}>
$$

where $D_{c}=\{x \in D: \Psi(x) \leq c\}$. By carrying out similar arguments as in the proof of Theorem 6.1 , we may deduce that

$$
\int_{D(c, 0)} k_{1}(x)|\bar{\partial} f|^{2} \leq 0
$$

The assumptions on $\Psi$ mean that $k_{1}$ is quasipositive on $D(c, 0)$. It follows that $f: D \rightarrow N$ is holomorphic.

Finally we give the following result:

Theorem 6.8. Let $M$ be as in Theorem 4.3 (resp. 4.7). Suppose $f: M \rightarrow N$ is either a pluriharmonic map into a Kähler manifold or a harmonic map into a Kähler manifold with strongly semi-negative curvature. If

$$
\liminf _{R \rightarrow \infty}\left(R \int_{\partial B_{\Phi}(R)}\left|\bar{\partial}_{b} f\right|^{2}\right)=0 \quad\left(\text { resp. } \liminf _{r \rightarrow \infty}\left(r \int_{\partial B_{r}\left(x_{0}\right)}\left|\bar{\partial}_{b} f\right|^{2}\right)=0\right)
$$

then $f: M \rightarrow N$ is holomorphic.

Proof. Without loss of generality, we assume that $M$ satisfies the conditions in Theorem 4.3. Set $X=\Phi \nabla \Phi$. For a regular value $R$ of $\Phi$, we have the unit outward normal vector field $\nu=\frac{\nabla \Phi}{|\nabla \Phi|}$ along $\partial B_{\Phi}(R)$. Using (6.4), we derive that

$$
\begin{aligned}
S_{\sigma}(X, \nu) & =R|\nabla \Phi|\left[\frac{|\sigma|^{2}}{2}-<\sigma(\nu), \sigma(\nu)>\right] \\
& \leq R \sqrt{k_{2}}\left|\bar{\partial}_{b} f\right|^{2}
\end{aligned}
$$


Likewise we have (6.2) on each $B_{\Phi}(R)$. By the assumption, there exists a sequence $\left\{R_{i}\right\}$ such that

$$
\lim _{i \rightarrow \infty} R_{i} \int_{\partial B_{r_{i}}\left(x_{0}\right)}\left|\bar{\partial}_{b} f\right|^{2}=0
$$

It follows that

$$
\int_{M} \lambda|\bar{\partial} f|^{2}=0
$$

where $\lambda$ is given by (4.4). Therefore $f$ is holomorphic.

Acknowledgments:The author would like to thank Professor J.G. Cao, Professor N. Mok, Professor M.C. Shaw, Professor S.W. Wei and Dr. Q.C. Ji for their valuable suggestions and helpful discussions. He would also like to thank Dr. H.Z. Lin and Dr. G.L. Yang for their careful reading of the manuscript.

\section{REFERENCES}

[Ba] P. Baird, Stress-energy tensors and the Lichnerowicz Laplacian, Journal of Geom. and Phys. 58 (2008), 1329-1342.

[BE] P. Baird, J. Eells, A conservation law for harmonic maps, Geometry Symposium, Utrecht 1980: Lecture notes in Mathematics, Vol. 894, Springer (1982), 1-25.

[Bo] S. Bochner, Analytic and meromorphic continuation by means of Green's formula, Ann. of Math., 44 (1943), 652-673.

[CL] J.Y. Chen, S.Y. Li, Holomorphic extensions of maps from the boundary of Kähler manifolds, Tôhoku Math. J. 49(1997), 585-597.

[DW] Y.X. Dong, S.W. Wei, On vanishing theorems for vector bundle valued p-forms and their applications, Comm. Math. Phys. Vol. 304 (2011), 329-368.

[EL] J. Eells, L. Lemaire, Selected topics in harmonic maps, CBMS Reg. Conf. Ser. Math. 50, Amer. Math. Soc., Providence, 1983.

[EF] J.F. Escober, A. Freire, The spectrum of the Laplacian of manifolds of positive curvature, T, Duke Math. J. Vol. 65, No.1 (1992) 1-21.

[EW] J. Eells and J. C. Wood, Harmonic maps from surfaces to complex projective spaces, Adv. in Math. 49 (1983) 217-263.

[GW] R.E. Greene, H. Wu, Function theory on manifolds which posses a pole, Lecture Notes in Math., Vol. 699, 1979, Springer-Verlag.

[Ha] R. Hamilton, Harmonic maps of manifolds with boundary, Lecture Notes in Math. 471, Springer-Verlag, Berlin-New York (1975).

[Ka] L. Karp, On Stokes' theorem for noncompact manifolds, Proc. A. M. S. Vol. 82 (1981) No. 3, 487-490.

[KW] H. Karcher, J.C. Wood, Non-existence results and growth properties for harmonic maps and forms, J. Reine Angew. Math. 353 (1984) 165-180.

[Li] P. Li, On the structure of complete Kähler manifolds with nonnegative curvature near infinity, Invent. Math. 99 (1990), 579-600.

[Mi] J. Milnor, Morse theory, Princeton University Press, 1963.

[NS] S. Nishikawa, K. Shiga, On the holomorphic equivalence of bounded domains in complete Kähler manifolds of nonpositive curvature, J. Math. Soc. Japan, Vol. 35(1983) No.2, 273278.

[OU] Y. Ohnita, S. Udagawa, Stability, complex-analyticity and constancy of pluriharmonic maps from compact Kaehler manifolds, Math. Z. 205 (1990), 629-644.

[PRS] S. Pigola, M. Rigoli, A. Setti, Vanishing and finiteness results in geometric analysis, Prog. in Math., Vol. 266, Birkhäuser, Basel·Boston·Berlin 2008. 
[Ra] J. Rawnsley, f-structures, f-twistor spaces and harmonic maps, Lecture Notes in Math. 1164, Springer, Berlin, 1985.

[RS] M. Rigoli, A.G. Setti, Liouville-type theorems for $\varphi$-subharmonic functions, Rev. Mat. Iberoamericana 17 (2001) 471-520.

[Sa] J. H. Sampson, Applications of harmonic maps to Kähler geometry, Complex differential geometry and nonlinear differential equations (Brunswick, Maine, 1984), 125-134, Contemp. Math. 49, Amer. Math. Soc., Providence, RI, 1986.

[Sc] R. Schoen, Existence and regularity for some geometric variational problems, Thesis, Stanford University (1977).

[Se] H.C.J. Sealey, The stress energy tensor and vanishing of $L^{2}$ harmonic forms, to appear.

[Sh] K. Shiga, An application of harmonic mapping to complex analytic geometry, Research Report of Gifu University, No. 17 (1981), 57-60.

[Si1] Y.T. Siu, The complex-analyticity of harmonic maps and the strong rigidity of compact Kähler manifolds, Ann. of Math. Vol.112 (1980) No.1, 73-111.

[Si2] Y.T. Siu, Complex-analyticity of harmonic maps, vanishing and Lefschetz theorems, J. Diff. Geom. Vol.17 (1982) 55-138.

[SY] Y. T. Siu, S. T. Yau, Compact Kahler manifolds of positive bisectional curvature, Invent. Math. 59 (1980) 184-204.

[Ta1] K. Takegoshi, A non-exisitence theorem for pluriharmonic maps of finite energy, Math. Z. 192, 21-27 (1986).

[Ta2] K. Takegoshi, Energy estimates and Liouville theorems for harmonic maps, Ann. Scient. Éc. Norm. Sup. 23 (1990) 563-592.

[To] D. Toledo, Rigidity Theorems in Kähler geometry and fundamental groups of varieties, Several Complex Variables, MSRI Publications, Volume 37(1999) 509-533.

[Ud] S. Udagawa, Holomorphicity of certain stable harmonic maps and minimal immersions, Proc. London Math. Soc. (3) 57 (1988), 577-598.

[Wa] J.M. Wan, Harmonic maps from $C^{n}$ to Kähler manifolds, Ph.D. thesis, Zhejiang Univ., 2010.

[Wo] J.C. Wood, An extension theorem for holomorphic mappings, Math. Proc. Camb. Phil. Soc. 88 (1980), 125-127.

[Wu] H. Wu, The Bochner technique in differential geometry, Mathematical Reports, Vol 3, Pt 2, Harwood Academic Publishing, London, 1987.

[Xi] Y.L. Xin, Differential forms, conservation law and monotonicity formula, Scientia Sinica (Ser A) Vol. XXIX (1986), 40-50.

Institute of Mathematics

Fudan University, Shanghai 200433

P.R. China

And

Key Laboratory of Mathematics

for Nonlinear Sciences

Ministry of Education

yxdong@fudan.edu.cn 